\title{
ECONOMICS
}

\section{Empirical evidence on the dynamics of investment under uncertainty in the U.S.}

by

\section{Qazi Haque}

Centre for Applied Macroeconomic Analysis

The University of Western Australia

\section{Leandro M. Magnusson}

Business School, The University of Western Australia

and

Kazuki Tomioka

University of Rochester 


\title{
Empirical evidence on the dynamics of investment under uncertainty in the U.S.*
}

\author{
Qazi Haque ${ }^{\dagger}$ \\ The University of Western Australia \\ Centre for Applied Macroeconomic Analysis \\ Leandro M. Magnusson ${ }^{\ddagger}$ \\ Kazuki Tomioka ${ }^{\S}$ \\ The University of Western Australia \\ University of Rochester
}

November 20, 2019

\begin{abstract}
We study the effects of financial uncertainty on investment dynamics in the U.S. using a vector autoregression with drifting parameters and stochastic volatilities. We find time-varying negative effects of financial uncertainty shocks on investment. These effects have declined in the post-WWII period but became more pronounced in the presence of the zero lower bound episode. We also find that the response of inflation to uncertainty shocks varies over time, and these shocks do not always act like aggregate demand shocks. Remarkably, the relevance of financial uncertainty shocks is found to be negligible during the Great Recession.
\end{abstract}

JEL Classifications: C11, C32, E22, E32, E44

Keywords: Uncertainty shocks; investment dynamics; TVP-VARs with stochastic volatility; Bayesian VARs; Great Recession.

${ }^{*}$ We would like to thank Guido Ascari, Ivan Alfaro, Giovanni Caggiano, Efrem Castelnuovo, Ken Clements, Michael Funke, Richard Heaney, Robert King, Sophocles Mavroeidis, Farhad Mohammad, Adrian Pagan, Aubrey Poon, Tom Simpson, Rod Tyers, Mark Weder, Francesco Zanetti and the seminar participants at The University of Western Australia, the Australian Conference for Economists 2017 (Sydney), the 26th Annual Society for Nonlinear Dynamics and Econometrics Symposium (Tokyo, 2018), the Australasian Meeting (Auckland, 2018) and the North American Summer Meeting (Seattle, 2019) of The Econometric Society. The authors acknowledge generous support from the Australian Research Council (DP170100697). Tomioka acknowledges financial support funded by the Commonwealth Government of Australia via the Research Training Program at The University of Western Australia. Declarations of interest: none.

${ }^{\dagger}$ CAMA and Department of Economics, Business School, The University of Western Australia. M251, 35 Stirling Hwy, Crawley WA 6009, Australia. Email: qazi.haque@uwa.edu.au.

${ }^{\ddagger}$ Department of Economics, Business School, The University of Western Australia. M251, 35 Stirling Hwy, Crawley WA 6009, Australia. Email: leandro.magnusson@uwa.edu.au (corresponding author).

${ }^{\S}$ Department of Economics, Harkness Hall, University of Rochester, Rochester, NY 14627. Email: ktomioka@ur.rochester.edu. 


\section{Introduction}

The recent global economic and financial crisis has led to a renewed interest in the relationship between uncertainty and the macroeconomy. ${ }^{1}$ Following the influential work by Bloom (2009), the role played by uncertainty shocks in driving macroeconomic fluctuations has been a key topic of research and discussion among both academics and policymakers. Part of the literature uses vector autoregression (VAR)-based analysis to identify the impact of uncertainty shocks. Recent contributions include Bloom (2009), Alexopoulos and Cohen (2009), Bachmann et al. (2013), Caggiano et al. (2014), Gilchrist et al. (2014), Baker et al. (2016), Leduc and Z. Liu (2016), Basu and Bundick (2017), Mumtaz and Theodoridis (2018) among others. In addition, a related literature study the transmission mechanism of uncertainty shocks from a theoretical standpoint. ${ }^{2}$ One consensus that has emerged on this topic is that (i) uncertainty shocks result in adverse effects on the economy (Bloom, 2009; Gilchrist et al., 2014; Jurado et al., 2015), and (ii) its effects are non-linear and time-varying (Caggiano et al., 2014; Mumtaz and Theodoridis, 2018).

First regarding (i), recent empirical studies support the notion that investment growth is retarded upon prevalence of uncertain environment, which produces a rapid drop in output and employment (Bloom, 2009). Part of the literature tends to attribute such observations to the "wait-and-see" behavior of firms arising from the real-options mechanism. Although the real-options mechanism gained overwhelming support since the seminal contribution of Bloom (2009), the channel by which uncertainty influences investment is not limited to it. For instance, Gilchrist et al. (2014) argue that the impact of uncertainty shocks on investment occurs primarily through its influence on financial markets. Moreover, Katayama and Kim (2018) argue that uncertainty shocks act as an adverse supply shock rather than a demand driven mechanism by documenting a negative relationship between the price and quantity of investment. Thus, how uncertainty affects economic activity and inflation is an open question in the literature.

Nevertheless, as pointed out by Benati (2016), the fact that a specific disturbance causes statistically significant decreases in real economic activity does not imply that such a shock plays an important role in either driving macroeconomic fluctuations on average

\footnotetext{
${ }^{1}$ See Castelnuovo (2019) for an extensive survey covering the recent literature on uncertainty.

${ }^{2}$ See Fernández-Villaverde et al. (2011), Christiano et al. (2014), Leduc and Z. Liu (2016), Basu and Bundick (2017), Bloom et al. (2018) for a non-exhaustive list.
} 
or during particular episodes. For instance, Benati (2016) argues that monetary policy shocks generate statistically significant response in real economic activity, but only play a marginal role in explaining the forecast error variances of macroeconomic aggregates and also generate statistically indistinguishable counterfactual paths.

Second regarding ii), employing a variety of different approaches, numerous studies have attempted to answer whether the effects of uncertainty on the economy has changed over time. For example, Caldara et al. (2016) compare the impulse responses of various real variables to uncertainty shocks derived from VARs. They show that the negative effects of uncertainty shock on real variables intensify when the sample period spans over the 2008-09 financial crisis, relative to when the sample period end prior to the onset of the financial crisis. Similarly, Caggiano et al. (2014) using a smooth-transition VAR show that the effects of uncertainty shocks are greater in magnitude during recessions. In contrast, Mumtaz and Theodoridis (2018), using a factor augmented VAR, argue that the negative impact of uncertainty shocks on real activity has gradually declined. Thus, although the literature appears to support that the effects of uncertainty shocks on real activity has changed over time, the nature of such time variation is still an open question.

In light of the discussion above, in this paper we use Bayesian time-varying parameter structural VAR with stochastic volatility to explore the role played by uncertainty in explaining the dynamics and evolution of aggregate investment. Following a large literature, our proxy for uncertainty is based on stock market volatility and hence our focus is on financial uncertainty. In principle, financial asset prices encompass all aspects of the firm's environment that investors view as important and therefore much research in this area use stock market volatility to infer fluctuations in uncertainty. ${ }^{3}$ We contribute to the literature on uncertainty by presenting and analyzing the posterior densities of several objects of interest that are relevant in shedding further light on the aforementioned questions. These include measures of uncertainty predictability and persistence, analyses of impulse response functions, and more crucially forecast error variance decompositions (FEVDs) and counterfactual simulations which together help to uncover the evolution of

\footnotetext{
${ }^{3}$ Other papers that also use stock market volatility to proxy for uncertainty include Romer (1990), Leahy and Whited (1996), Greasley and Madsen (2006), Bloom et al. (2007), Bloom (2009), Gilchrist et al. (2014), and Basu and Bundick (2017) among others. Alternative proxies for uncertainty have been constructed using forecast disagreement (Bachmann et al., 2013), real GDP forecast errors (B. Rossi and Sekhposyan, 2015), forecast errors of several macroeconomic and financial indicators (Jurado et al., 2015; Ludvigson et al., 2018), Bloomberg forecasts (Scotti, 2016), interest rate data (Mumtaz and Zanetti, 2013; Istrefi and Mouabbi, 2018), policy uncertainty (Baker et al., 2016) and Google Trends data (Castelnuovo and Tran, 2017).
} 
the dynamic relationship between investment and uncertainty. Main findings of the paper can be summarized as follows.

First, we document substantial time variation in the volatility of uncertainty shocks and the predictability and persistence of uncertainty, measured as the deviation of uncertainty from its long run component. We find the volatility of uncertainty shocks to increase during major economic, financial and political shocks, followed by rising uncertainty predictability and persistence.

Second, we confirm that uncertainty shocks are contractionary shocks resulting in a statistically significant decline in investment. However, the "volatility overshoot" effect of uncertainty shock, namely the rebound in real activity following the initial decline which is consistent with the "wait-and-see" behavior of investment documented by Bloom (2009), is only evident since the 1990s. Interestingly, the "volatility overshoot" effect coincides with a larger decline in the real interest rate, which suggests that the recovery of investment may be partly driven by endogenously expansionary monetary policy as suggested by Bachmann et al. (2013).

Third, we find that the co-movement between investment growth and inflation conditional on uncertainty shocks has varied over time, being mostly negative in the period between the 1960s and the mid-1980s and turning positive only since the 2000s. These results show that uncertainty shocks do not always act as aggregate "demand shocks", unlike the suggestion of Leduc and Z. Liu (2016) and Basu and Bundick (2017). Instead, the positive response of inflation to uncertainty shock in the first part of the sample is consistent with the findings of Mumtaz and Theodoridis (2018) and supports the existence of pricing bias channel postulated by Ferníndez-Villaverde et al. (2015). 4

Fourth, the impact of uncertainty shocks on investment growth has declined over time, which is in line with the findings of Mumtaz and Theodoridis (2018). However, unlike these authors, we also find a systematic decline in the magnitude of the response of inflation and the real interest rate to uncertainty shocks. The positive response of inflation has become more muted over time, eventually turning negative in the early 2000s, and at the same time the real interest rate has turned more negative. One possible explanation for these findings, highlighted by Mumtaz and Theodoridis (2018), is an increase in the

\footnotetext{
${ }^{4}$ The pricing bias channel implies that firms subject to price stickiness have the incentive to set prices above the level they would target in the absence of uncertainty in order to avoid losing profits in case favorable economic conditions realize in the future (Ferníndez-Villaverde et al. (2015)).
} 
responsiveness of interest rates to inflation over time. A stronger response to inflation makes price-setting firms less forward looking and, therefore, less concerned about future inflation. This in turn allows the monetary authority to cut back interest rates more quickly, thereby dampening the negative effects of uncertainty shocks on real activity.

Fifth, we find a change in the impact of uncertainty shocks when we include the zero lower bound (ZLB) episode affecting conventional monetary policy in our estimation. In particular, the response of investment growth to uncertainty shock becomes slightly more negative while the real interest rate response becomes less negative. This finding is consistent with a number of studies suggesting that the impact of uncertainty shocks might be more pronounced when the ZLB binds (Johannsen, 2014; Basu and Bundick, 2017; Caggiano et al., 2017).

Sixth, despite the statistically significant impact of uncertainty shocks on macroeconomic variables as evident from VAR impulse responses, such shocks play only a marginal role in explaining the variance of macroeconomic aggregates. Furthermore, counterfactual simulations suggest that the evolution of these variables are statistically indistinguishable from the actual series when we shut down the identified uncertainty shocks. Absent such shocks during the Great Recession, investment growth would have deviated away from the actual only in 2008Q4. As for inflation and interest rate, the difference between actual and counterfactual path is indistinguishable. Therefore, as pointed out by Benati (2016), the fact that a particular shock generates statistically significant impulse responses does not necessarily imply that this shock has played an important role in driving macroeconomic fluctuations. Overall, these results provide limited evidence that financial uncertainty shocks, as identified by unanticipated changes in stock market volatility, may have played an important role during the Great Recession.

The papers most closely related to ours are studies by Benati (2016) and Mumtaz and Theodoridis (2018). Benati (2016) employs a Bayesian structural VAR with stochastic volatility to study the role played by policy uncertainty shocks during the Great Recession in the U.S., the Euro area, the U.K. and Canada, and the Great Depression in the U.S. Using several alternative identification strategies, the paper finds that policy uncertainty shocks played a marginal role during both episodes in the Euro area, the U.K. and Canada, and during the Great Depression in the U.S. However, policy uncertainty shocks played a statistically significant and economically non-negligible role for the U.S. during the Great 
Recession and shows that absent policy uncertainty shocks the U.S. unemployment rate would have been about 2 percentage points higher during and after the Great Recession. In comparison, the current paper looks at the effects of financial uncertainty shocks based on stock market volatility, which has served as a popular proxy for uncertainty since the seminal contribution of Bloom (2009). Moreover, using a similar Bayesian structural VAR with stochastic volatility but also allowing for time-varying parameters, we document time-varying impact of such shocks on the economy, with a particular focus on investment.

Mumtaz and Theodoridis (2018) investigate the changing transmission of uncertainty shocks on the U.S. economy using a factor augmented VAR (FAVAR) model that allows for the estimation of a measure of uncertainty which encompasses volatility from the real and financial sectors of the economy and serve as a proxy for "macroeconomic" uncertainty. Their proposed FAVAR also contains time-varying parameters and provides an estimate of the time-varying response of macroeconomic variables to shocks to this uncertainty measure. One key feature of the specification of Mumtaz and Theodoridis (2018) is that the volatility of macroeconomic uncertainty shocks is allowed to have an impact on the VAR's dynamics, thus introducing a link between second and first moments. Their results suggest that the impact of macroeconomic uncertainty shocks on real activity has declined systematically over time. In contrast, they find that the response of inflation and interest rates to this shock has remained fairly stable. The current paper, on the other hand, employs a standard time-varying parameter VAR with stochastic volatility to study the dynamics of financial uncertainty shocks. While overall macroeconomic uncertainty is clearly of interest from a policymaking standpoint, nevertheless studying the time-varying effects of stock market volatility is also interesting on its own right, particularly since a large portion of the literature has used such measure as a proxy for uncertainty. Another motivation for studying the effects of financial uncertainty shocks is that policy might react differently to movements in asset market conditions than to movements in uncertainty picked up by other measures as suggested by Bachmann et al. (2013). Indeed, we find time variation in the impact of financial uncertainty shocks not only for investment, but also for inflation and real interest rates. Unlike Mumtaz and Theodoridis (2018), we also find that the presence of the ZLB has affected the impact of stock market volatility shocks on the economy. Moreover, the current paper also looks at the FEVDs and conducts counterfactual simulations to asses the role played by such shocks during the Great Recession. Therefore, our contribution may be seen as complementary to Benati (2016) and Mumtaz 
and Theodoridis (2018).

The next section outlines the econometric framework, the data used in the estimation and the identification strategy. Section 3 presents the results from the baseline model. Robustness checks are performed in Section 4. Section 5 provides evidence on the relative importance of uncertainty shocks in terms of explaining the forecast error variances while Section 6 conducts counterfactual simulations to uncover the role played by these shocks during the Great Recession. Finally, Section 7 concludes the paper. Further empirical results along with a detailed description of the methodology and estimation techniques are in the online Supplement.

\section{Econometric framework}

Our primary objective is to empirically investigate potentially time-varying relationship between investment and uncertainty. One way to achieve this is to use a VAR with timevarying parameters and stochastic volatility. This section describes such a VAR along with the estimation methodology, the variables included in the VAR, and the identification strategy.

\subsection{Time-varying VAR with stochastic volatility}

A reduced form time-varying VAR of order $p$ is expressed as

$$
\mathbf{y}_{t}=\mathbf{c}_{t}+\sum_{p=1}^{P} \mathbf{B}_{p, t} \mathbf{y}_{t-p}+\mathbf{u}_{t}, \quad \mathbf{u}_{t} \sim \mathrm{N}\left(\mathbf{0}, \boldsymbol{\Omega}_{t}\right),
$$

where $\mathbf{y}_{t}$ is an $n \times 1$ vector of endogenous variables, $\mathbf{c}_{t}$ is an $n \times 1$ column vector of intercepts, $\mathbf{B}_{p, t}$ is an $n \times n$ matrix containing the $p^{t h}$ lag autoregressive coefficients, and $\mathbf{u}_{t}$ are heteroscedastic unobservable shocks with variance covariance matrix $\boldsymbol{\Omega}_{t}$.

This multivariate time-varying VAR has several advantages over the single equation modeling traditionally used in estimating investment models. First, our hypothesis implies that modeling the time dimension of the relationship between investment and uncertainty is essential. Second, the model enables us to identify and distinguish internal system and external shocks, which can further be decomposed with a set of identifying assumptions. Such decomposition allows us to observe less direct relationship between the variables, not 
achievable in univariate regressions.

\subsection{Model specification and estimation}

With reference to equation (1), we form a vector $\boldsymbol{\beta}_{t}$ by stacking the elements of $\mathbf{c}_{t}$ and $\mathbf{B}_{p, t}$ equation by equation such that $\boldsymbol{\beta}_{t} \equiv \operatorname{vec}\left(\left[\mathbf{c}_{t}, \mathbf{B}_{1, t}, \cdots, \mathbf{B}_{P, t}\right]^{\prime}\right)$ and let $\mathbf{X}_{t} \equiv \mathbf{I}_{n} \otimes$ $\left(1, \mathbf{y}_{t-1}^{\prime}, \cdots, \mathbf{y}_{t-P}^{\prime}\right)$, where $\otimes$ denotes the Kronecker product. Then equation (1) can be recast as a measurement equation of the state space model

$$
\mathbf{y}_{t}=\mathbf{X}_{t} \boldsymbol{\beta}_{t}+\mathbf{A}_{t}^{-1} \boldsymbol{\Sigma}_{t} \boldsymbol{\epsilon}_{t}, \quad t=P+1, \cdots, T
$$

where $\mathbf{A}_{t}^{-1}$ is a lower triangular, time-varying matrix with ones along the main diagonal, $\boldsymbol{\Sigma}_{t}$ is a diagonal, time-varying matrix comprising of standard deviations of the structural shocks, and $\boldsymbol{\epsilon}_{t} \sim \mathrm{N}\left(\mathbf{0}, \mathbf{I}_{n}\right)$ is a vector of standardized structural shocks. Note that the variance-covariance matrix of reduced form disturbance term, $\boldsymbol{\Omega}_{t}$, is factored $\boldsymbol{\Omega}_{t}=\mathbf{A}_{t}^{-1} \boldsymbol{\Sigma}_{t} \boldsymbol{\Sigma}_{t}^{\prime}\left(\mathbf{A}_{t}^{-1}\right)^{\prime}$, where $\mathbf{A}_{t}^{-1}$ and $\boldsymbol{\Sigma}_{t}$, are respectively defined as

$$
\mathbf{A}_{t}^{-1} \equiv\left[\begin{array}{cccc}
1 & 0 & \cdots & 0 \\
\widetilde{a}_{21, t} & 1 & \ddots & \vdots \\
\vdots & \ddots & \ddots & 0 \\
\widetilde{a}_{n 1, t} & \cdots & \widetilde{a}_{n n-1, t} & 1
\end{array}\right], \quad \boldsymbol{\Sigma}_{t} \equiv\left[\begin{array}{cccc}
\sigma_{1, t} & 0 & \cdots & 0 \\
0 & \ddots & \ddots & \vdots \\
\vdots & \ddots & \ddots & 0 \\
0 & \cdots & 0 & \sigma_{n, t}
\end{array}\right] .
$$

Note that lower triangular elements of $\mathbf{A}_{t}^{-1}$ are subscripted with $t$, implying that the covariance parameters in $\boldsymbol{\Omega}_{t}$ are all time varying. Thus the time-varying VAR we consider is of the form presented in Primiceri (2005) and differs from that in Cogley and Sargent (2005) as they model covariance parameters as static parameters.

For future references, we represent equation (2) in a companion form

$$
\mathbf{z}_{t}=\mathbf{v}_{t}+\mathbf{M}_{t} \mathbf{z}_{t-1}+\mathbf{P}_{t} \boldsymbol{\epsilon}_{t}
$$

where $\mathbf{z}_{t}$ is a vector containing endogenous variables up to the lag order of $P-1, \mathbf{v}_{t}$ comprises the intercept term, $\mathbf{M}_{t}$ is the companion matrix, and $\mathbf{P}_{t}$ is a Cholesky matrix that factors the companion form of the reduced form variance-covariance matrix, $\mathbf{V}_{t}=$ $\mathbf{P}_{t} \mathbf{P}_{t}^{\prime}$ 
The dynamics of the model's time-varying parameters are specified as follows:

$$
\boldsymbol{\beta}_{t+1}=\boldsymbol{\beta}_{t}+\boldsymbol{v}_{t}, \quad \boldsymbol{\alpha}_{t+1}=\boldsymbol{\alpha}_{t}+\zeta_{t}, \quad \log \boldsymbol{\sigma}_{t+1}^{2}=\log \boldsymbol{\sigma}_{t}^{2}+\boldsymbol{\eta}_{t},
$$

where $\boldsymbol{\beta}_{t}$ comprises of stacked reduced form coefficients and follows a random walk process, $\boldsymbol{\alpha}_{t}$ is a vector of non-zero and non-unitary elements of $\mathbf{A}_{t}$ which are stacked by rows, $\boldsymbol{\alpha}_{t}=\left(a_{21, t}, \cdots, a_{n n-1, t}\right)^{\prime}$ and also follows a random walk process, and $\boldsymbol{\sigma}_{t}^{2}$ represents the vector of the leading diagonal elements of $\boldsymbol{\Sigma}_{t} \boldsymbol{\Sigma}_{t}^{\prime}$ such that $\boldsymbol{\sigma}_{t}^{2}=\left(\sigma_{1, t}^{2}, \cdots, \sigma_{n, t}^{2}\right)^{\prime}$ and follows a geometric random walk process. Next, we make a distributional assumption on $\boldsymbol{\epsilon}_{t}, \boldsymbol{v}_{t}$, $\boldsymbol{\zeta}_{t}$ and $\boldsymbol{\eta}_{t}$, which we now vectorize as $\boldsymbol{\psi}=\left(\boldsymbol{\epsilon}_{t}, \boldsymbol{v}_{t}, \boldsymbol{\zeta}_{t}, \boldsymbol{\eta}_{t}\right)^{\prime}$. Following Primiceri (2005), we assume $\boldsymbol{\psi}$ to be jointly Gaussian with mutually uncorrelated white noise, zero mean and variances defined by $\mathbf{I}_{n}$ and the hyper-parameters $\mathbf{Q}, \mathbf{S}$ and $\mathbf{W}$, such that $\boldsymbol{\psi} \sim$ $\mathbf{N}\left[\mathbf{0}, \operatorname{diag}\left(\mathbf{I}_{n}, \mathbf{Q}, \mathbf{S}, \mathbf{W}\right)\right]$, where $\mathbf{I}_{n}$ is a $n$-dimensional identity matrix, and $\mathbf{Q}, \mathbf{S}$ and $\mathbf{W}$ are all diagonal positive semi-definite matrices. ${ }^{5}$

For the initial states of parameters, we place priors that are considered uninformative for this class of models. Specifically, $\boldsymbol{\beta}_{P+1} \sim \mathrm{N}(\mathbf{0}, 10 \mathbf{I}), \boldsymbol{\alpha}_{P+1} \sim \mathrm{N}(\mathbf{0}, 10 \mathbf{I})$ and $\log \boldsymbol{\sigma}_{P+1}^{2} \sim \mathbf{N}(\mathbf{0}, 10 \mathbf{I})$. The corresponding hyper-parameters' priors are respectively $Q_{i} \sim$ $\mathrm{G}^{-1}(40 / 2,0.004 / 2), S_{i} \sim \mathrm{G}^{-1}(12 / 2,0.01 / 2), W_{i} \sim \mathrm{G}^{-1}(12 / 2,0.01 / 2)$, where $\mathrm{G}^{-1}$ denotes the inverse Gamma distribution, and $Q_{i}, S_{i}$ and $W_{i}$ denote respective diagonal elements in $\mathbf{Q}$, $\mathbf{S}$ and $\mathbf{W}$. Essentially, the hyper-parameters in the time-varying VAR governs the rate at which the parameters vary over time. ${ }^{6}$

The time-varying VAR is then estimated by the Markov chain Monte Carlo (MCMC) routine developed by Nakajima (2011) with one important modification as discussed below. We employ Nakajima (2011)'s routine over Primiceri (2005)'s as it allows for more efficient, joint sampling of the parameters $\mathbf{b}^{T}=\left\{\boldsymbol{\beta}_{t}\right\}_{t=P+1}^{T}$ and $\mathbf{a}^{T}=\left\{\boldsymbol{\alpha}_{t}\right\}_{t=P+1}^{T}$ via the simulation smoother of de Jong and Shephard (1995). The block sampler of Shephard and Pitts (1997) (algorithm corrected by Watanabe and Omori (2004)) is used to sample the stochastic

\footnotetext{
${ }^{5}$ The diagonal assumption of $\mathbf{S}$ implies that the non-zero and non-unitary elements of the matrix $\mathbf{A}_{t}$ are assumed to evolve and hence be distributed independently across equations, but correlated within equations. We further assume $\mathbf{Q}$ and $\mathbf{W}$ to be diagonal matrices to economize on the estimation of parameters, given reports that suggest that these cross-equation restrictions are of minimal importance (Primiceri, 2005; Nakajima, 2011).

${ }^{6}$ Note that priors set over the hyper-parameters for the time-varying VAR coefficients are more tighter/informative than the ones set for variance and covaraince states. We set these priors on the hyper-parameters given Sims (2001)'s comment on Cogley and Sargent (2001)'s work (where they modeled the variances as static parameters) that part of the time variation in the variances of the model was absorbed by VAR coefficients, thus overstating the drifts in the systematic relationship between the endogenous variables.
} 
volatility, $\mathbf{h}^{T}=\left\{\log \boldsymbol{\sigma}_{t}\right\}_{t=P+1}^{T}$. Hyper-parameters of these parameters are then generated from the inverse Gamma distribution, conditional on their respective parameters.

Our modification of Nakajima (2011)'s MCMC routine takes the form of adding the rejection step as in Cogley and Sargent (2005). Specifically, after simulating the unrestricted posterior, we solve for the eigenvalues of the characteristic equation, $\lambda_{t}$, and reject draws that produce $\max \left(\left|\boldsymbol{\lambda}_{t}\right|\right) \geq 1$, such that $\boldsymbol{\lambda}_{t}$ are all inside the unit circle. This rejection step ensures that our posterior is restricted to comprise only the draws that produce stable VAR coefficients at every point in time. ${ }^{7}$ Employing this MCMC routine, we collect 10,000 restricted posterior samples for posterior analyses, noting that we discard the first 2,000 draws to ensure the convergence of the chain. Interested readers are referred to the online Supplement for the details of the MCMC routine and the evaluation of the convergence of the posterior samples.

\subsection{Data and identification strategy}

Our analysis depends on the vector $\mathbf{y}_{t}=\left[s_{t}, \pi_{t}, i_{t}, r_{t}\right]^{\prime}$, where, $s_{t}$ is the uncertainty proxy, $\pi_{t}$ stands for the annualized quarter-on-quarter inflation rate, $i_{t}$ is the annualized quarter-on-quarter growth rate of investment, and $r_{t}$ is the real interest rate. We use quarterly time series data for the U.S. over the period 1962Q3 to 2018Q4, 1962Q3 being the first available quarter for the uncertainty index . Here, we briefly outline the dataset used for producing the baseline results and relegate the details to the online Supplement.

We use the 30-day implied volatility of the S\&P100, VXO, as our proxy for uncertainty, which we demean and standardize over our sample period. The Chicago Board of Options Exchange VXO index is considered as a forward-looking measure of uncertainty since it reflects market-based expectations about returns on the underlying security. This index is unavailable before 1986. Following Bloom (2009), pre-1986 monthly return volatilities are computed by employing the monthly standard deviation of the daily S\&P500 index normalized to the same mean and variance as the VXO index when they overlap from 1986 onward. ${ }^{8}$ Inflation is calculated as the annualized quarter-on-quarter percentage growth

\footnotetext{
${ }^{7}$ See Appendix B of Cogley and Sargent (2005) for the justification of this step.

${ }^{8}$ In the online Supplement, we construct a measure of uncertainty by extracting the first principal component (FPC) from three different proxies: (i) stock market volatility as described here, (ii) the proxy estimated by Gilchrist et al. (2014) which captures common shocks in the idiosyncratic volatility of equity returns, and (iii) the financial uncertainty index proposed by Jurado et al. (2015) which measures a common component in the time-varying volatilities of h-step ahead forecast errors across a large number of financial
} 
rate of the implicit GDP deflator. Our measure of investment follows that of Justiniano et al. (2010) and is the sum of personal consumption expenditure on durable goods and gross private domestic investment, both in per capita terms and deflated to 2012 dollars using their respective implicit deflators. Finally, the ex-post real interest rate is used as a proxy for the ex-ante real interest rate and is the difference between the Federal Funds rate and the annualized GDP deflator inflation rate. Quarterly observations of monthly data are constructed via quarterly averaging. Furthermore, as our sample spans over the period when the Federal Funds rate was constrained by the zero lower bound (ZLB), we replace the Federal Funds rate with the shadow rate proposed by Wu and Xia (2016) during that period.

We employ a recursive identification scheme to identify uncertainty shocks based on the Cholesky factorization of the reduced form residual's variance-covariance matrix as a mapping between the reduced form and the structural model. This is consistent with previous VAR literature such as Bloom (2009), Caggiano et al. (2014), Jurado et al. (2015), and Basu and Bundick (2017) among others, that identify uncertainty shocks based on timing assumptions.

As we rely on the recursive identification scheme to orthogonalize the VAR innovations, the order by which the endogenous variables enter the VAR matters. The conventional wisdom is to enter the most endogenous variable last, such that more endogenous variables affect less endogenous variables with a lag. To this end, we rely on previous VAR studies that analyze the macroeconomic dynamics of uncertainty shocks and treat uncertainty as the most exogenous process and thus order it first. This implies that contemporaneous macroeconomic effects by our identified uncertainty shocks are allowed. The real interest rate is ordered last so as to best capture the behavior and the reaction of the Federal Reserve (Fed). Thus, the mechanism here is such that private agents making investment decisions are able to immediately change their decision given changes in uncertainty and the monetary authority is allowed to respond accordingly. Note that ordering the VXO first in our vector attributes all the one-step ahead forecast error in the VXO to uncertainty shocks. Therefore, our results should be interpreted as providing an upper bound on the effects of uncertainty shocks. ${ }^{9}$

series. The uncertainty proxy based on stock market volatility is very similar to the FPC with a correlation of 0.95 .

${ }^{9}$ Ordering uncertainty first is consistent with the effects of uncertainty shocks in the theoretical New Keynesian model developed by Basu and Bundick (2017). 


\section{Results}

Using the ordering and priors presented in Section 2, we estimate the time-varying VAR with two lags. The lag length is set in accord with Cogley and Sargent (2005) and Primiceri (2005). The results from this baseline specification are presented in this section while the next section conducts checks of robustness with different specifications of the model.

\subsection{Volatility, predictability and persistence of uncertainty}

We start our analyses by first examining the evolution of the second moments of uncertainty. Figure 1 plots the median and $68 \%$ credible intervals of the posterior distributions of the standard deviations of the estimated uncertainty shocks volatility. This volatility appears to increase during major economic, financial and political shocks as shown in this figure.

Figure 1: Volatility of uncertainty shocks

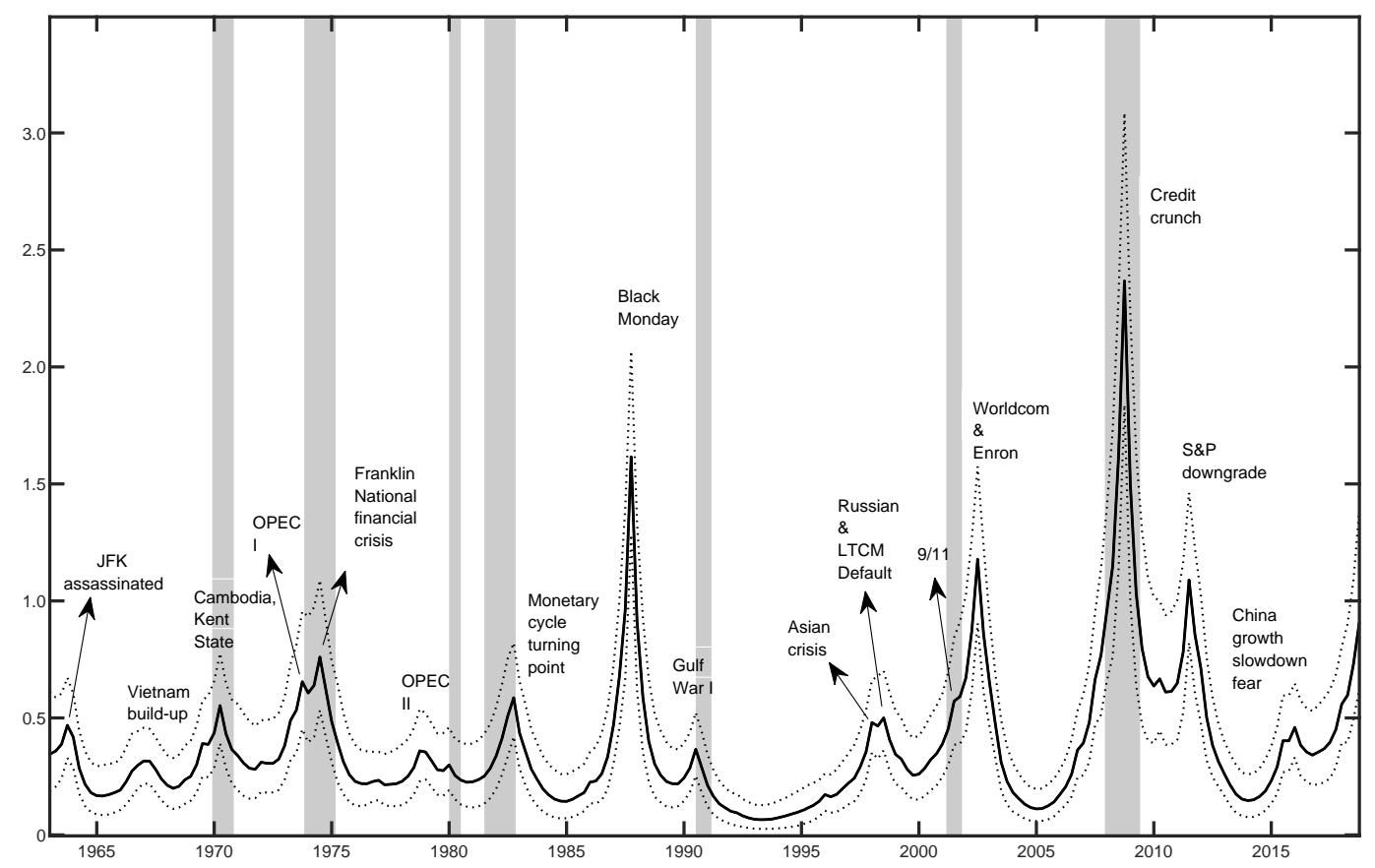

Note: Posterior median and $68 \%$ credible intervals of the estimated standard deviations of uncertainty shocks. Vertical gray-shaded regions in the background depict NBER recessions.

We are also interested in studying the (time-varying) predictability and persistence of uncertainty and document its relationship with uncertainty shock volatility. The measure 
of predictability is defined as one minus the ratio of conditional variance over unconditional variance

$$
R_{s, t}^{2}(H)=1-\frac{\mathbf{k}_{s}\left[\sum_{h=0}^{H-1} \boldsymbol{\Phi}_{h, t} \boldsymbol{\Omega}_{t} \boldsymbol{\Phi}_{h, t}^{\prime}\right] \mathbf{k}_{s}^{\prime}}{\mathbf{k}_{s}\left[\sum_{h=0}^{\infty} \boldsymbol{\Phi}_{h, t} \boldsymbol{\Omega}_{t} \boldsymbol{\Phi}_{h, t}^{\prime}\right] \mathbf{k}_{s}^{\prime}},
$$

where $\mathbf{k}_{s}$ is a selection (row) vector for uncertainty proxy, $\boldsymbol{\Phi}_{h, t}$ is the $h^{\text {th }}$ matrix lag of the corresponding vector moving average $(\operatorname{VMA}(\infty))$ form of equation $(1),{ }^{10}$ and $\boldsymbol{\Omega}_{t}$ is the variance-covariance matrix as defined before.

A statistic related to predictability is the persistence of a variable, measured via the normalized spectrum on the frequency domain. The statistic is defined as the ratio of the spectrum of a variable over the unconditional variance

$$
g_{s, t}(\omega)=\frac{f_{s, t}(\omega)}{\int_{-\pi}^{\pi} f_{s, t}(\omega) \mathrm{d} \omega},
$$

where

$$
f_{s, t}(\omega)=\mathbf{k}_{s}\left(\boldsymbol{\Upsilon}_{t}^{-1}\right) \frac{\boldsymbol{\Omega}_{t}}{2 \pi}\left(\boldsymbol{\Upsilon}_{t}^{-1}\right)^{*} \mathbf{k}_{s}^{\prime}
$$

Here, $\mathbf{k}_{s}$ is a row vector selecting uncertainty, $\left(\mathbf{\Upsilon}_{t}^{-1}\right)^{*}$ denotes the conjugate transpose of the inverse of $\boldsymbol{\Upsilon}_{t}=\mathbf{I}_{n}-\mathbf{\Phi}_{h, t} \exp (-i \omega)$ for $h=1$, and $\boldsymbol{\Omega}_{t}$ is a variance-covariance matrix.

We follow Benati and Surico (2008) and interpret the statistic proposed by Cogley et al. (2010) at forecast horizon $H$ as the measure of predictability and the statistic by Cogley and Sargent (2005) as the measure of persistence at frequency $\omega^{11}$

The top row of Figure 2 portrays 3-dimensional plots of the posterior median of predictability and persistence estimates. Directly below it are their respective posterior median estimates at $H=1$ and $\omega=0$ together with $68 \%$ credible bands. There is substantial time variations in the predictability and the persistence of uncertainty. However, as seen in the bottom panels of Figure 2, the persistence of uncertainty is less precisely estimated than the predictability measure. ${ }^{12}$

\footnotetext{
${ }^{10}$ Note that such rewriting is assured by the satisfaction of the stability condition, achieved by the rejection of the draws that produce explosive roots in the MCMC.

${ }^{11}$ Note that both statistics, by construction, measure uncertainty 'gap' predictability and persistence, away from the unconditional mean, i.e., the long-run component. Note moreover, that in a multivariate context, the mapping between the two statistics are not one-for-one unlike in the univariate case (Benati and Surico, 2008). Thus, whether these related statistics reveal different features of uncertainty is worth investigating.

${ }^{12}$ This last finding coincides with the trend inflation literature. For instance, estimates reported by Cogley and Sargent $(2001,2005)$ suggest a decline in inflation gap persistence, but the estimates are imprecise and statistically insignificant. In fact, Cogley et al. (2010) came up with the statistic based on predictability in order to obtain more precise estimates which show a statistically significant decline in inflation gap persistence.
} 
Figure 2: Predictability and persistence of uncertainty
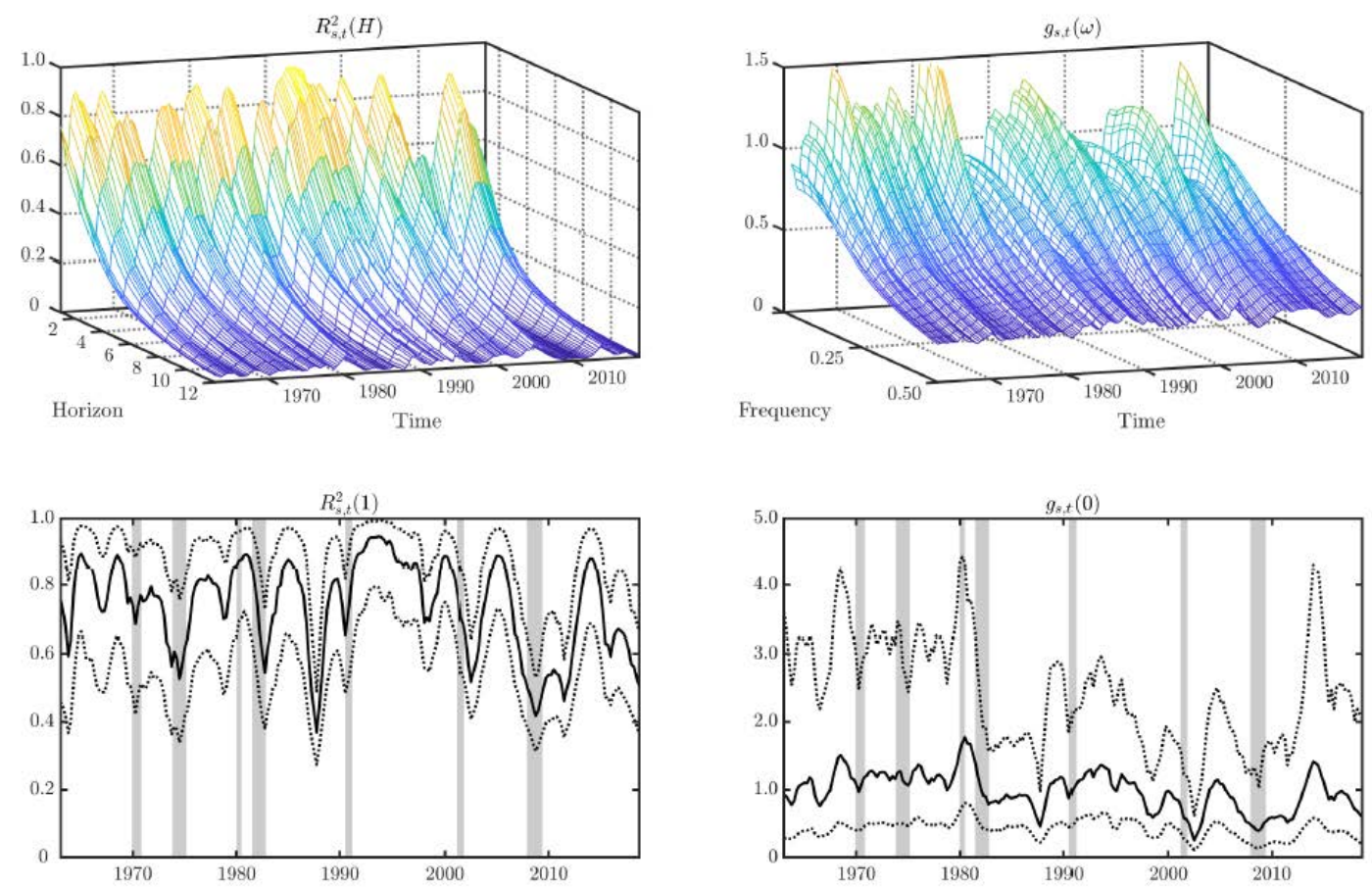

Note: 3D plots depict the posterior median of predictability and persistence over different frequencies and horizon respectively. The bottom row depicts the posterior median and $68 \%$ credible intervals at $H=1$ for predictability and $\omega=0$ for persistence. Vertical gray-shaded regions depict NBER recessions.

Digging deeper, Figure 3 shows that the predictability of uncertainty is negatively correlated with uncertainty shock volatility. ${ }^{13}$ Since recessions, as identified by the NBER, and other major economic, financial and political shocks are associated with spikes in uncertainty, this explains why such periods are mostly followed by a rise of predictability and persistence. This is more evident since the 2000s, with an increase in both predictability and persistence after the 2001 recession, and again from the Great Recession onwards.

\subsection{Evolution of the impact of uncertainty shocks on investment}

Figure 4 shows the median and $68 \%$ credible intervals of the posterior distributions of the impulse response functions (IRFs) of uncertainty, inflation, investment growth and the real interest rate to a normalized uncertainty shock on impact and at the 1-year and 2year ahead horizons. To allow comparability between the computed impulse responses over

\footnotetext{
${ }^{13}$ To a lesser extent, this is also true for the persistence measure.
} 
Figure 3: Uncertainty predictability and shock volatility

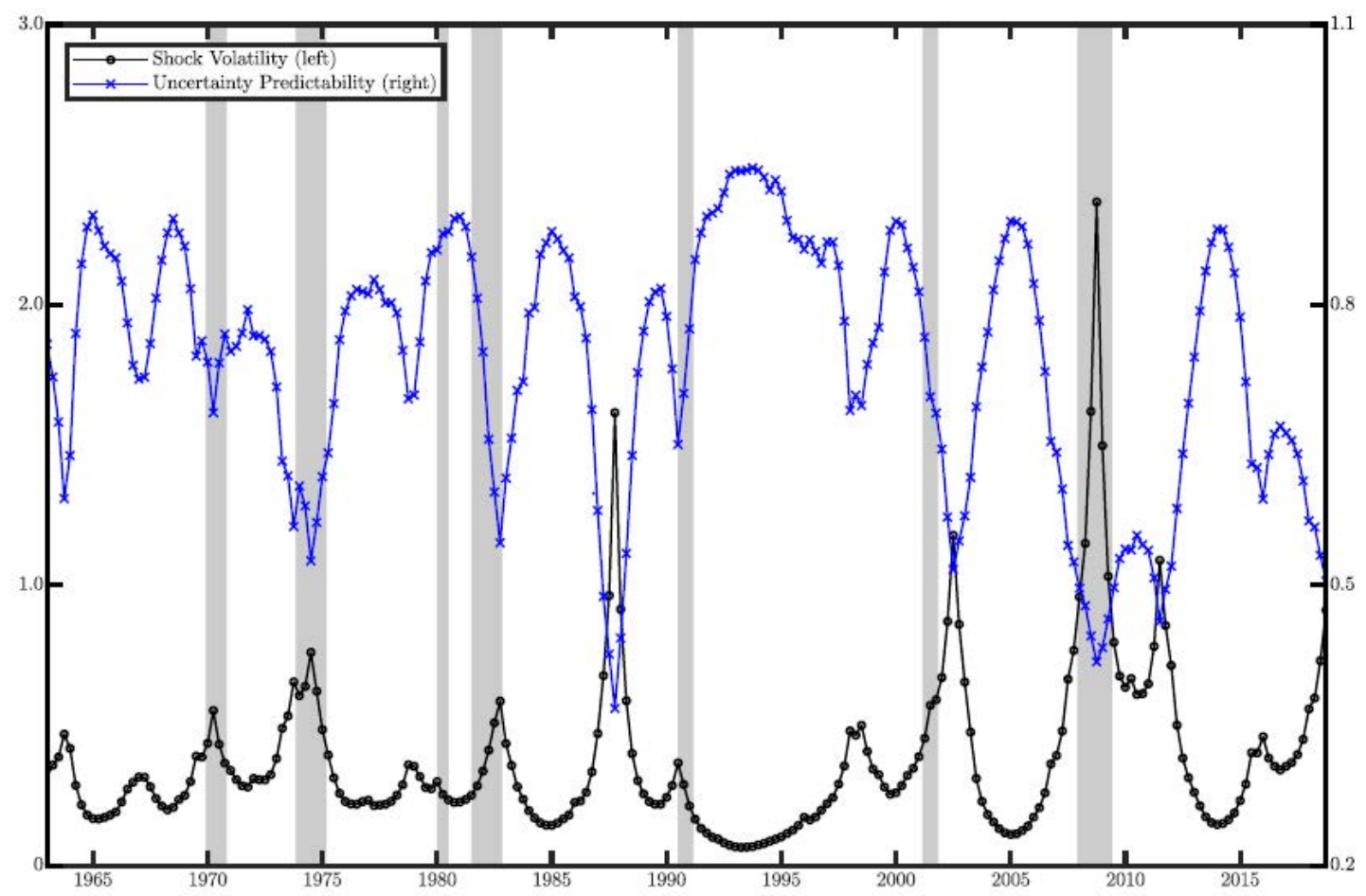

Note: Posterior median of uncertainty predictability and shock volatility. Vertical gray-shaded regions in the background depict NBER recessions.

time, we apply in each period the time series average of stochastic volatility for uncertainty, $\bar{\sigma}_{s}=1 /(T-P) \sum_{t=P+1}^{T} \exp \left(\log \sigma_{s, t}^{2} / 2\right)$. Thus, the impulse responses reported here depict the responses of variables when an average-sized shock strikes the VAR system. Several notable findings arise.

First, consistent with previous studies, we confirm that uncertainty shocks are contractionary shocks that aggravate economic conditions. Investment growth declines significantly and follows a hump-shaped path before going back to its steady state value within about one year. ${ }^{14}$ The "volatility overshoot" effect of uncertainty shock, that is the rebound in real activity following the initial decline after a positive uncertainty shock consistent with the "wait-and-see" optimal behavior of investment (Bloom, 2009), is only evident since the 1990s. Interestingly, such "drop, rebound, and overshoot" effect coincides with a larger decline in the real interest rate following an uncertainty shock. This finding

\footnotetext{
${ }^{14}$ The hump-shaped impulse response of investment growth to uncertainty shock can be clearly seen when the impulse responses are plotted over horizons as opposed to over time, see Figure S.2 in the Supplement.
} 
Figure 4: Impulse responses to a normalized uncertainty shock
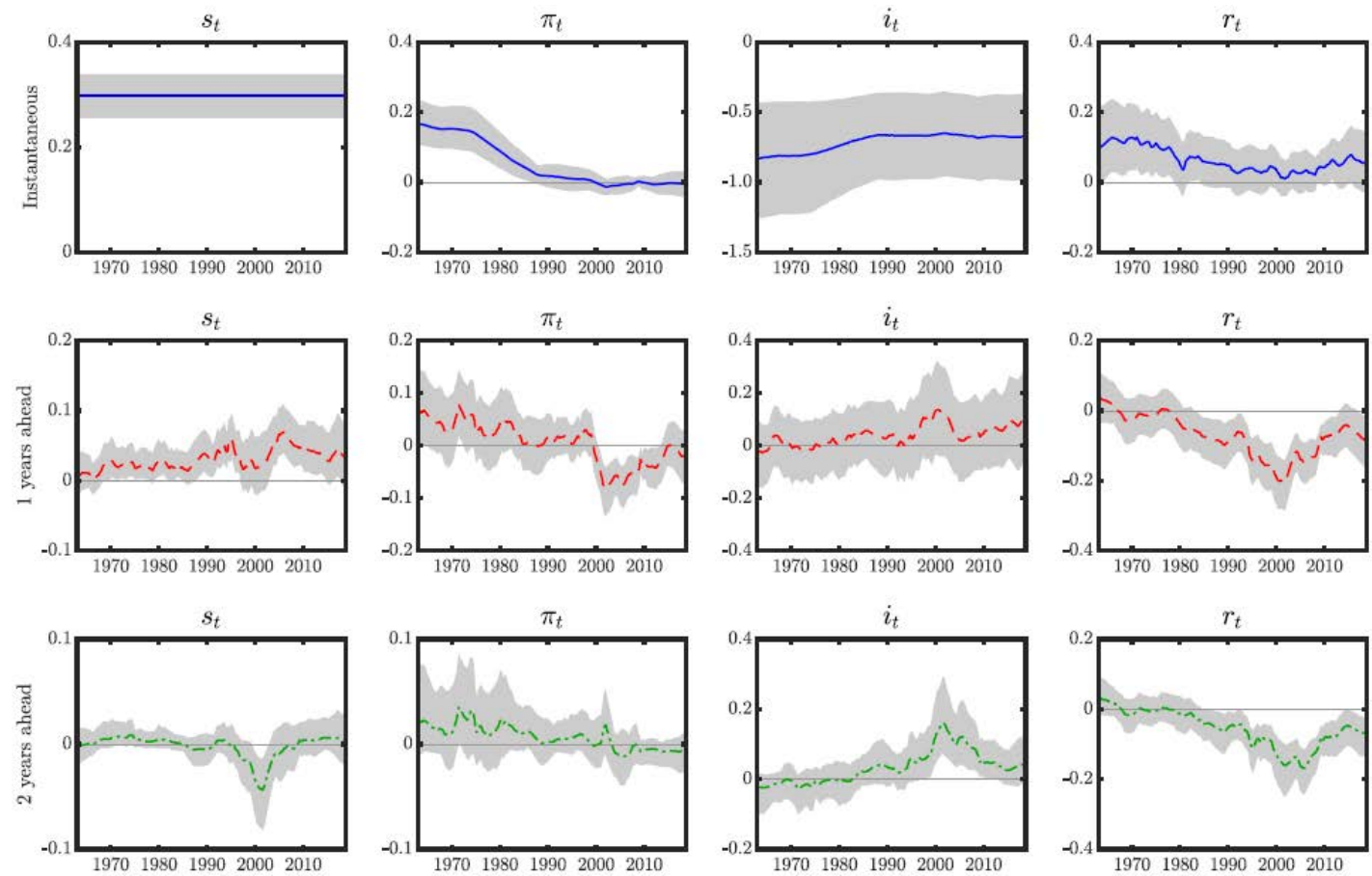

Note: Instantaneous, 1-year and 2-year ahead impulse responses. The baseline identification ordering $\mathbf{y}_{t}=\left[s_{t}, \pi_{t}, i_{t}, r_{t}\right]^{\prime}$ adopted. The first column shows the response of $s_{t}$ : uncertainty, second column shows the response of $\pi_{t}$ : inflation, third column shows the response of $i_{t}$ : investment growth and the fourth column shows the response of $r_{t}$ : real interest rate. The gray shaded regions represent the $68 \%$ posterior credible intervals around the posterior median depicted in solid, dashed, and dashed-dotted lines.

is suggestive that the rebound effect may be partly driven by endogenously expansionary monetary policy as argued by Bachmann et al. (2013).

Conditional on uncertainty shocks, we find a negative co-movement between investment growth and inflation until about the mid-1980s, a statistically insignificant response of inflation between the mid-1980s and the 2000s, and finally a decline in inflation thereafter. These results differ with those obtained by Basu and Bundick (2017) and Leduc and Z. Liu (2016), who suggest that uncertainty shocks act as aggregate "demand" shocks in the sense that these shocks result in a decline of both activity and inflation. ${ }^{15}$ Rather, the positive response of inflation to uncertainty shock is in line with the empirical findings of Mumtaz and Theodoridis (2018) and supports the existence of pricing bias channel postulated in Ferníndez-Villaverde et al. (2015).

\footnotetext{
${ }^{15}$ Fasani and L. Rossi (2018) show that Leduc and Z. Liu (2016)'s model prediction on inflation can be overturned by modeling interest rate inertia.
} 
Figure 5: Accumulated impulse responses to a normalized uncertainty shock
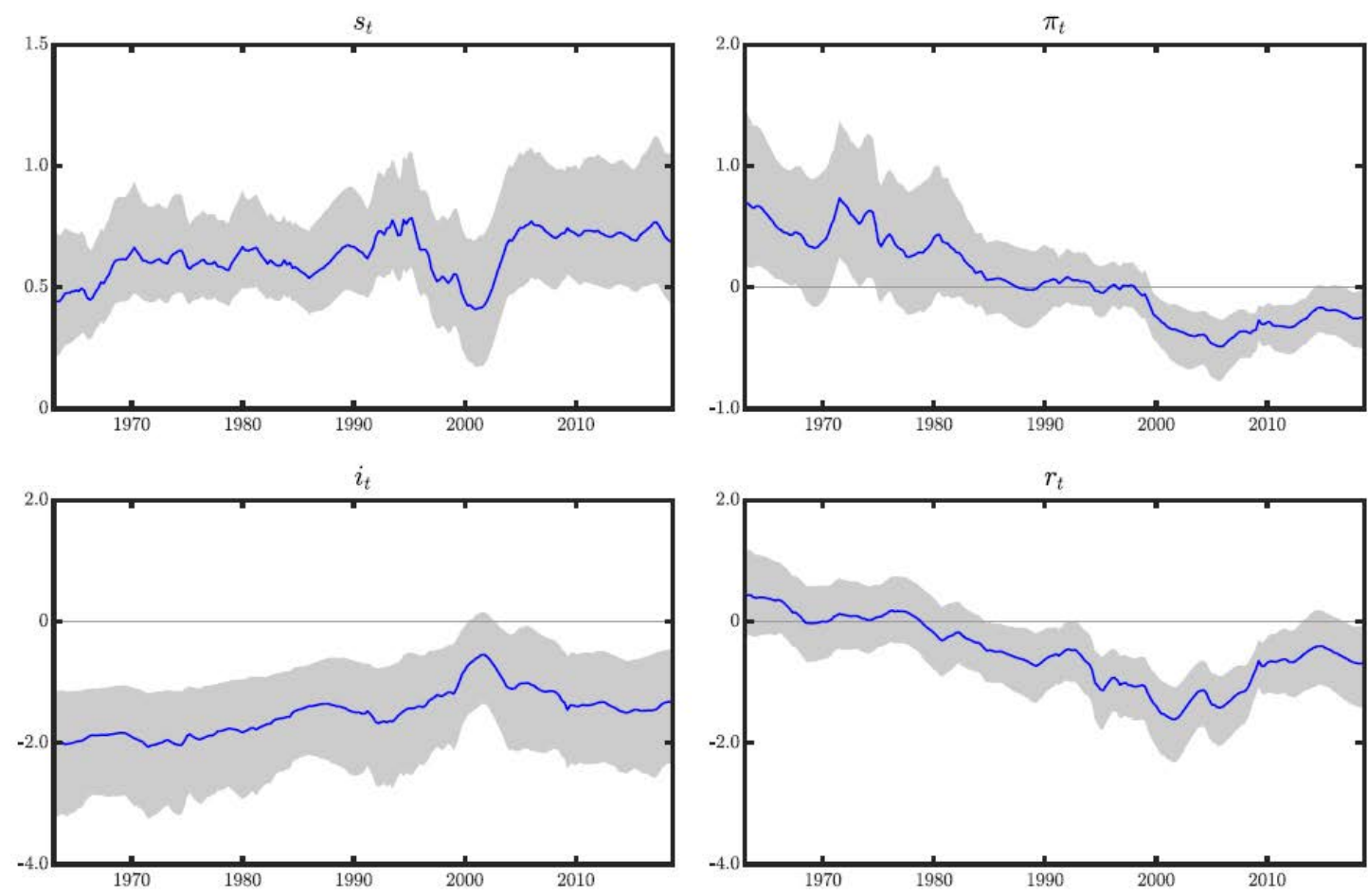

Note: Accumulated response at the 3-year horizon; sample period 1962Q3-2018Q4.

Figure 5 plots the cumulated impulse responses at the 3 -year horizon and document more clearly the time variation in the response of the variables to an average-sized uncertainty shock. ${ }^{16}$ The figure shows that the response of investment growth to uncertainty shock has declined over time. For instance, investment growth fell by around $2 \%$ during the 1960s and the early 1970s. In contrast, the decline in investment growth is closer to around $0.6 \%$ in the early 2000s. This finding is in line with Mumtaz and Theodoridis (2018) who also report similar decline in the impact of uncertainty shock on real activity. However, in contrast to Mumtaz and Theodoridis (2018), we also find a systematic decline in the magnitude of the response of inflation and real interest rate till early to mid-2000s. In particular, the positive response of inflation became more muted over time, eventually turning negative around the turn of the century. During the same time period, the response of real interest rate turned more negative.

One possible explanation for these changes may be structural shifts as highlighted in Fernández-Villaverde and Rubio-Ramírez (2008), which points to an increase in the Federal

\footnotetext{
${ }^{16}$ The focus on 3 -year horizon is for simplicity, but the results are robust to alternative horizons.
} 
Reserve's anti-inflationary stance. ${ }^{17}$ As shown by Mumtaz and Theodoridis (2018), an increase in the responsiveness of interest rates to inflation implies that inflation responds less to uncertainty shocks. This is because agents become less concerned about expected inflation, which in turn allows the central bank to cut back interest rates more quickly and aggressively, thereby dampening the adverse effect of uncertainty shocks on real activity. A stronger response to inflation makes price-setting firms less forward looking. The resulting decrease in firms' pricing bias can explain the decline in the positive response of inflation and the eventual switch to a negative response following an uncertainty shock.

The accumulated impulse responses shown in Figure 5 also suggest a change in the impact of uncertainty shocks in the mid-to-late 2000s. Following an uncertainty shock, the response of investment growth becomes slightly more negative while that of both inflation and the real interest rate become less negative. One possible explanation for this finding may be due to the zero lower bound (ZLB) affecting conventional monetary policy. The Federal Reserve hit the ZLB in December 2008 and has maintained the Fed Funds rate at historically low levels for several years afterwards.

A number of studies suggest that the impact of uncertainty shocks might be more pronounced when the ZLB binds (Johannsen, 2014; Basu and Bundick, 2017; Caggiano et al., 2017). To bolster this argument, we then estimate the model over the period 1962Q32008Q3, which excludes the ZLB period and the years afterwards. Figure 6 shows the cumulated impulse responses at the 3-year horizon as before. In the absence of the ZLB episode, the responses of inflation, investment growth and the real interest rate almost monotonically dampen over time, which is in line with a stronger response to inflation. In other words, we do not find any switch in the cumulated impulse responses in the 2000s.

Our results therefore suggest that the presence of the ZLB may indeed magnify the macroeconomic effects of uncertainty shocks. ${ }^{18}$ This result is interesting given that we replace the Federal Funds rate with the shadow rate of Wu and Xia (2016) for the period when short term interest rate was constrained by the ZLB. One implication is that quantitative easing and other unconventional monetary policies conducted by the Federal Reserve, to the extent captured by the shadow rate of $\mathrm{Wu}$ and Xia (2016), is not as

\footnotetext{
${ }^{17}$ Several studies offer robust evidence regarding changes in the policy reaction function, see Clarida et al. (2000), Lubik and Schorfheide (2004), Davig and Leeper (2007), Haque et al. (2018), Haque (2019) among others.

${ }^{18}$ This evidence is also in line with recent research studying the effects of first-moment macroeconomic shocks in presence of the zero lower bound, see P. Liu et al. (2019).
} 
Figure 6: Accumulated impulse responses to a normalized uncertainty shock that excludes the ZLB period
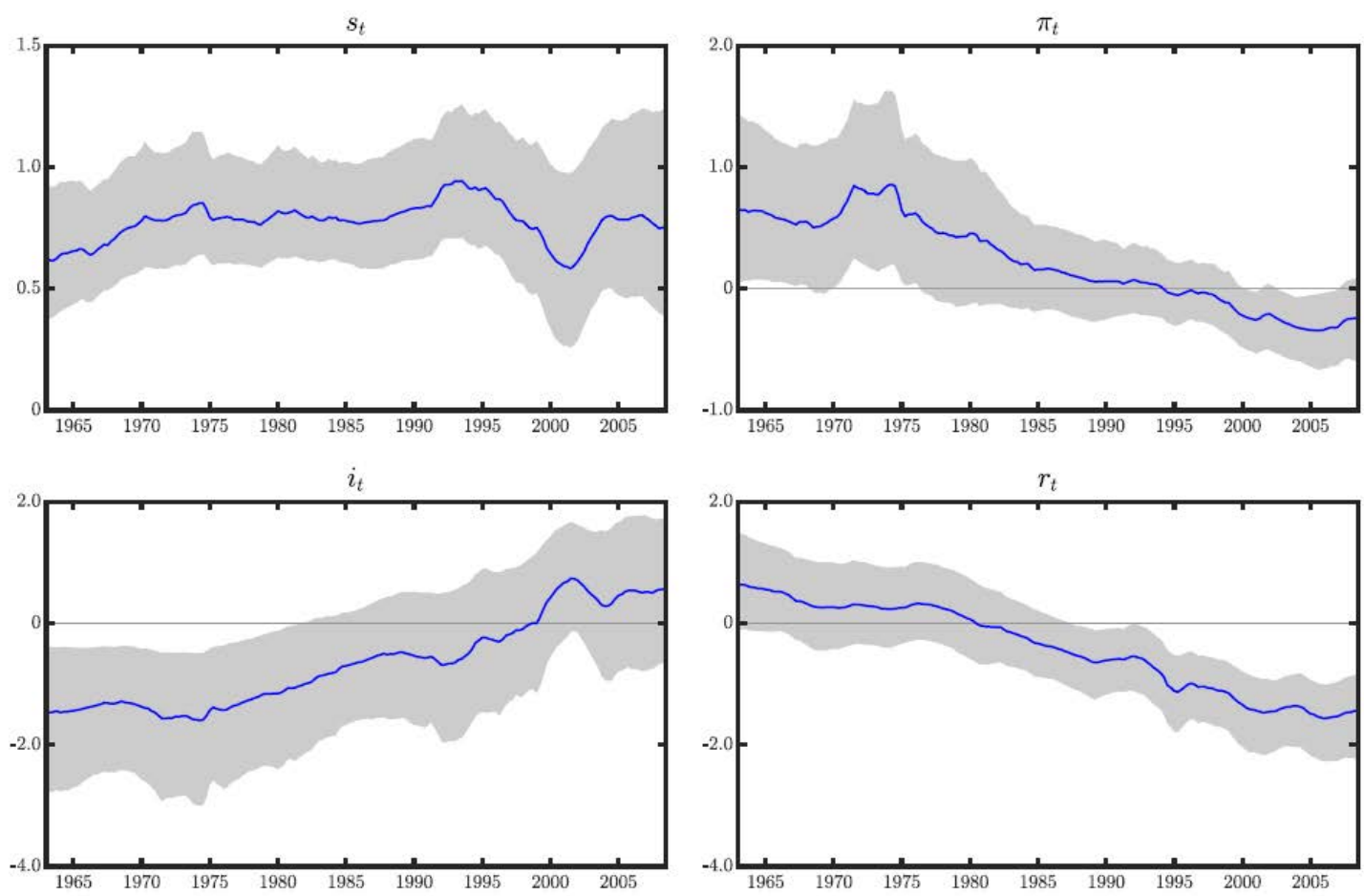

Note: Accumulated response at the 3-year horizon; sample period 1962Q3-2008Q3.

effective as conventional monetary policy in offsetting the negative effects of uncertainty shocks on investment growth. To check this argument, we also estimate the model without replacing the Federal Funds rate with the shadow rate during the ZLB episode and our results remain essentially unchanged.

\section{Robustness checks}

Based on the results derived in the previous section, it is evident that the dynamics of investment, inflation and interest rates have changed over time. However, it is possible that these findings are biased due to misspecification of the VAR model. For instance, if the VAR does not include sufficient information to correctly estimate the impact of uncertainty shocks, the impulse responses could be biased. Another issue is that our benchmark analysis focuses only on a particular measure of investment. Therefore, in this section, we conduct several robustness checks with respect to omitted variables and 
alternative measures of investment and activity indicator.

\subsection{S\&P100}

In the baseline analysis, we identify uncertainty shocks as exogenous movements of the VXO, which capture the volatility of the stock market. However, variations in the level of the stock market are negatively correlated with the VXO and may be an important driver of macroeconomic aggregates. Our baseline model might capture variations in the level of the stock market as variations in uncertainty. As a result, following Caggiano et al. (2014), we estimate a five-variate VAR $\mathbf{y}_{t}=\left[S \& P 100_{t}, s_{t}, \pi_{t}, i_{t}, r_{t}\right]^{\prime}$, where S\&P100 capture the level of the stock market. ${ }^{19}$

\subsection{Consumer sentiment}

Consumer sentiment might be another important driver of the economy as it contains information concerning agents' expectations over the future state of the economy and, therefore, might also include anticipated effects of uncertainty shocks. Therefore, we estimate a five-variate VAR $\mathbf{y}_{t}=\left[\operatorname{sent}_{t}, s_{t}, \pi_{t}, i_{t}, r_{t}\right]^{\prime}$, where "sent" stands for consumer sentiment and is the index of consumer expectations based on information collected via the Michigan Survey of Consumers. ${ }^{20}$ Moreover, this measure of consumer sentiment is (negatively) correlated with the VXO, implying that the baseline model might mix up confidence shocks and uncertainty shocks.

\subsection{Credit spread}

Gilchrist et al. (2014) question the relevance of the "wait-and-see" channel of uncertainty and argue that financial distortions are the main mechanism through which fluctuations in uncertainty affect macroeconomic outcomes. ${ }^{21}$ In particular, they suggest that the impact of uncertainty on investment occurs primarily through changes in the credit spreads. To

\footnotetext{
${ }^{19}$ Since S\&P100 exhibit a clear upward trend, we estimate the VAR using detrended data obtained by applying Hodrick-Prescott filter to the log of the S\&P100 index with a smoothing parameter of 1600.

${ }^{20}$ This index is calculated as an average of expectations about business conditions over the next year, expectations about aggregate business conditions over the next five years and expectations about personal financial conditions over the next year.

${ }^{21}$ Financial frictions have also been shown to be important for the propagation of other shocks, for example, news shocks as in Görtz et al. (2016).
} 
allow for uncertainty shocks to operate through credit spreads, we estimate a five-variate VAR $\mathbf{y}_{t}=\left[s_{t}, \text { spread }_{t}, \pi_{t}, i_{t}, r_{t}\right]^{\prime}$, where "spread" is the corporate bond spread computed as the difference between the Baa 30-year yield and the 30-year Treasury bond yield following Bachmann et al. (2013). ${ }^{22}$ We also consider the implications of uncertainty shocks conditional on the information contained in the spread.

\subsection{Alternative macroeconomic activity indicators}

In the baseline analysis, we measure investment as the sum of personal consumption expenditure on durable goods and gross private domestic investment. While being a popular measure of investment in the literature (see Justiniano et al. (2010)), this investment series is more volatile and procyclical and matters for the importance of different shocks in explaining the variability of macroeconomic aggregates. For instance, in an estimated medium-scale DSGE model, Justiniano et al. (2010) argue that investment shocks turn out to be the major driver of business cycles when investment is measured in this way. To ensure that our results are not driven by this particular measure, we also estimate the model using fixed private investment as a measure of investment following Smets and Wouters (2007). Additionally, we also estimate the model using real gross domestic product as a measure of economic activity instead of investment.

The outcome of all these robustness checks are reported in Figure 7. In almost all cases, the nature of time variation in the macroeconomic aggregates is comparable to the baseline model. Admittedly, some quantitative effects are present. For instance, the VAR controlling for movements in the S\&P100 index features a milder and smoother response of investment growth to uncertainty shocks. Likewise, similar milder and smoother response is observed when using real GDP instead of investment in the VAR, which is somewhat a-priori expected since real GDP is less volatile than investment. The response of inflation and the real interest rate turn out to be quite robust across scenarios.

Finally. we also check the robustness of our results with respect to the priors, lag lengths and ordering of the variables in the VAR. Our results remain unchanged and are delegated to the Supplement.

\footnotetext{
${ }^{22}$ In periods when the 30 -year bond is missing we use the 20-year treasury bond yield as in Bachmann et al. (2013).
} 
Figure 7: Robustness checks
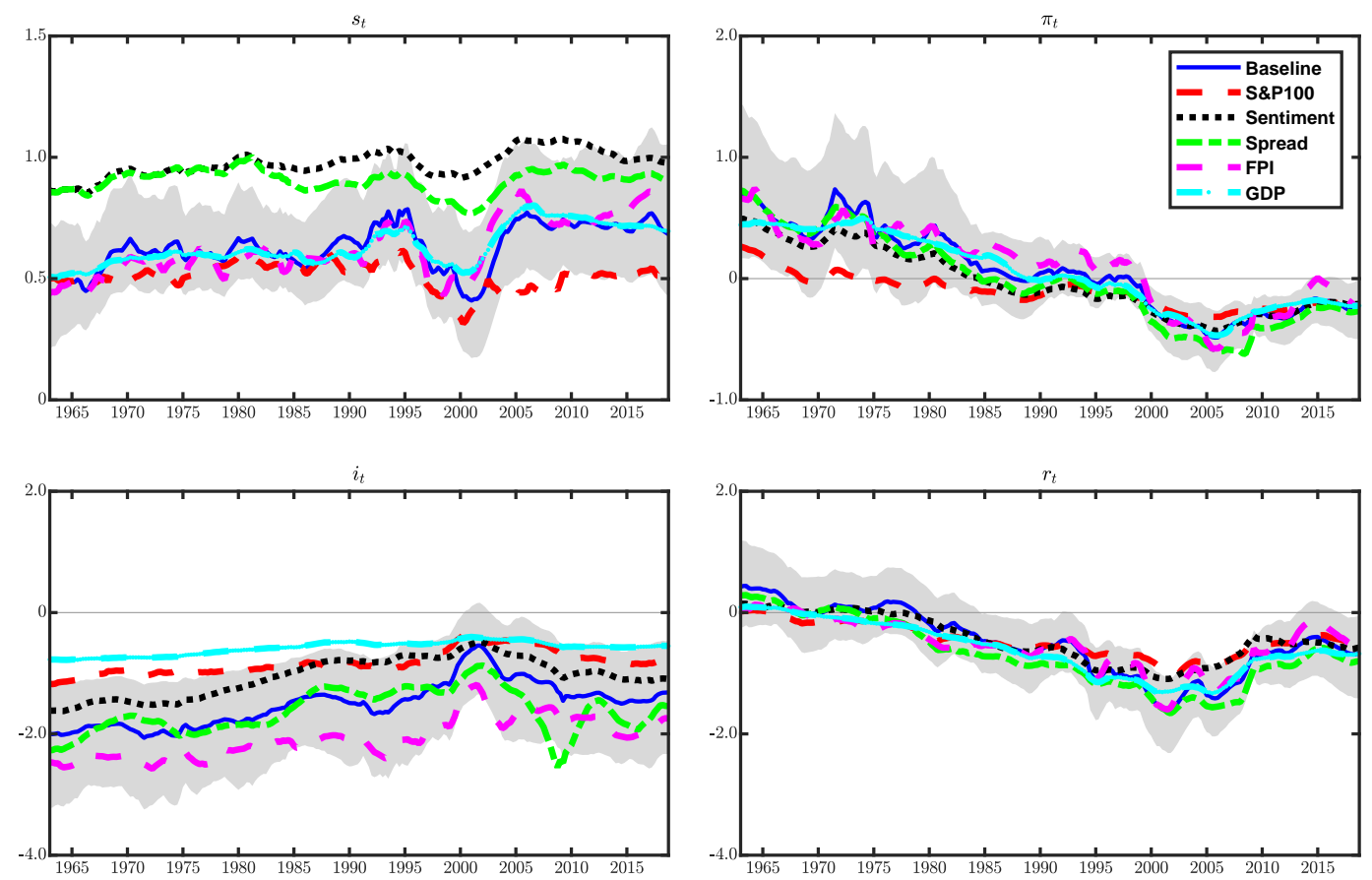

Note: Macroeconomic effects to an average-sized shock to VXO. Sample: 1962Q3-2018Q4. Gray shaded area corresponds to $68 \%$ credible interval from the baseline model.

\section{Forecast error variance decompositions}

Figure 8 shows the medians and $68 \%$ credible intervals of the posterior distributions of the fractions of the 1-year and 2-year ahead forecast error variances (FEVs) of the variables explained by uncertainty shocks. The forecast error variance decompositions (FEVDs) exhibit noticeable variations over time with large spikes occurring during major shocks such as the October 1987 stock market crash, the dot-com crash, and the credit crunch during the GFC. Uncertainty shocks during those periods are estimated to be responsible for an important share of the variance of inflation, investment growth and the real interest rate while mostly playing only a marginal role at other times. Moreover, uncertainty shocks play a significant role in driving the evolution of the VXO index itself.

The above FEVD analysis suggest that uncertainty shocks might have played an important role in the evolution of macroeconomic aggregates in certain episodes including the GFC. However, one potential issue is that the estimated contribution of uncertainty shocks to the FEVs of macroeconomic variables might be biased due to lack of relevant 
Figure 8: Forecast error variance decomposition - baseline model

1-year horizon
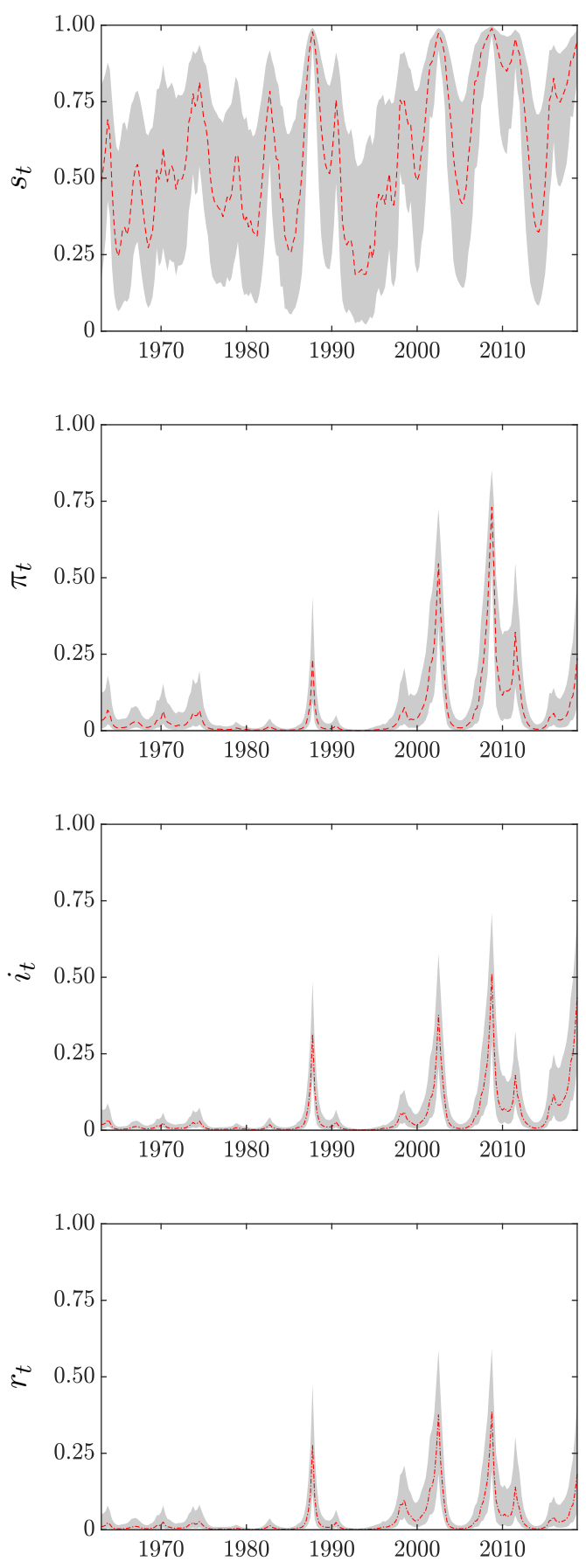

2-year horizon
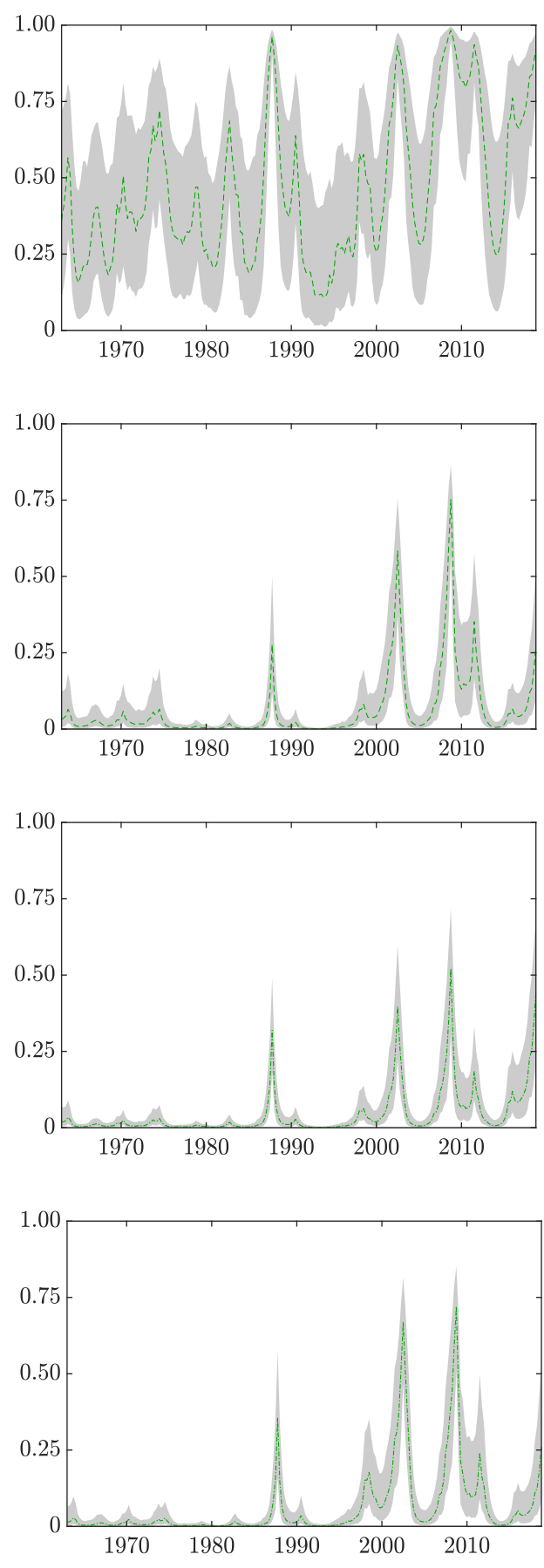

Note: FEVDs at 1-year and 2-year horizons.

information in our baseline VAR. In particular, the estimated spikes in the FEVDs coincide with large variations in the level of the stock market and so the baseline model might mix 
up variations in uncertainty with variations in the level of the stock market index.

To illustrate this point, Figure 9 plots the contribution of uncertainty shocks conditional on a five-variate VAR including S\&P100. ${ }^{23}$ As seen in the figure, results based on this five-variate VAR point toward a uniformly negligible role of uncertainty shocks in explaining fluctuations in inflation, investment growth and the real interest rate. For instance, the median FEVDs are uniformly below $5 \%$ for the entire sample period. As before, uncertainty shocks continue to play an important role in driving the evolution of the VXO index. These results are in line with the findings of Jurado et al. (2015) who also find a limited role played by VXO shocks.

\section{Counterfactual simulations}

Given that the sensitivity of investment growth to uncertainty shock has increased (in the negative direction) since the financial crisis, we conduct a counterfactual simulation over the financial crisis and the subsequent period. To this end, we consider a counterfactual scenario in which we mute the identified uncertainty shocks by setting it to zero. Figure 10 shows the actual historical paths of $\mathrm{VXO}$, inflation, investment growth, and the real interest rate, along with the medians and the $68 \%$ credible intervals of the posterior distributions of the counterfactual paths simulated by "shutting down" the identified uncertainty shock from 2008Q1 onward for each draw from the posterior.

Observing Figure 10, there are two main periods when uncertainty shocks play a nonnegligible role and the counterfactual level of uncertainty turns out to be lower than the actual path. The first period occurs in the fourth quarter of 2008, when the uncertainty proxy peaks during the onset of the financial crisis. It is evident that, in the counterfactual scenario, the rise in the uncertainty proxy is not as extreme. Such phenomenon occurs again in the third quarter of 2011 when the US government bonds were downgraded by Standard \& Poor's (S\&P). The financial market reaction has been negative and the S\&P500 lost 14\% over the quarter, which is the largest loss second to the financial crisis. Nevertheless, investment growth appears to deviate away from the actual path only during 2008Q4, reaching a median trough of $-24.9 \%$ as opposed to the actual trough of $-38.7 \%$. As for inflation and the real interest rate, the differences between actual and counterfactual

\footnotetext{
${ }^{23}$ The order of the VAR is the same as in the robustness section where $\mathbf{y}_{t}=\left[S \& P 100_{t}, s_{t}, \pi_{t}, i_{t}, r_{t}\right]$. That is, we look at the FEVDs of uncertainty shocks conditional on the level of the stock market.
} 
Figure 9: Forecast error variance decomposition - alternative model with S\&P100 index

1-year horizon
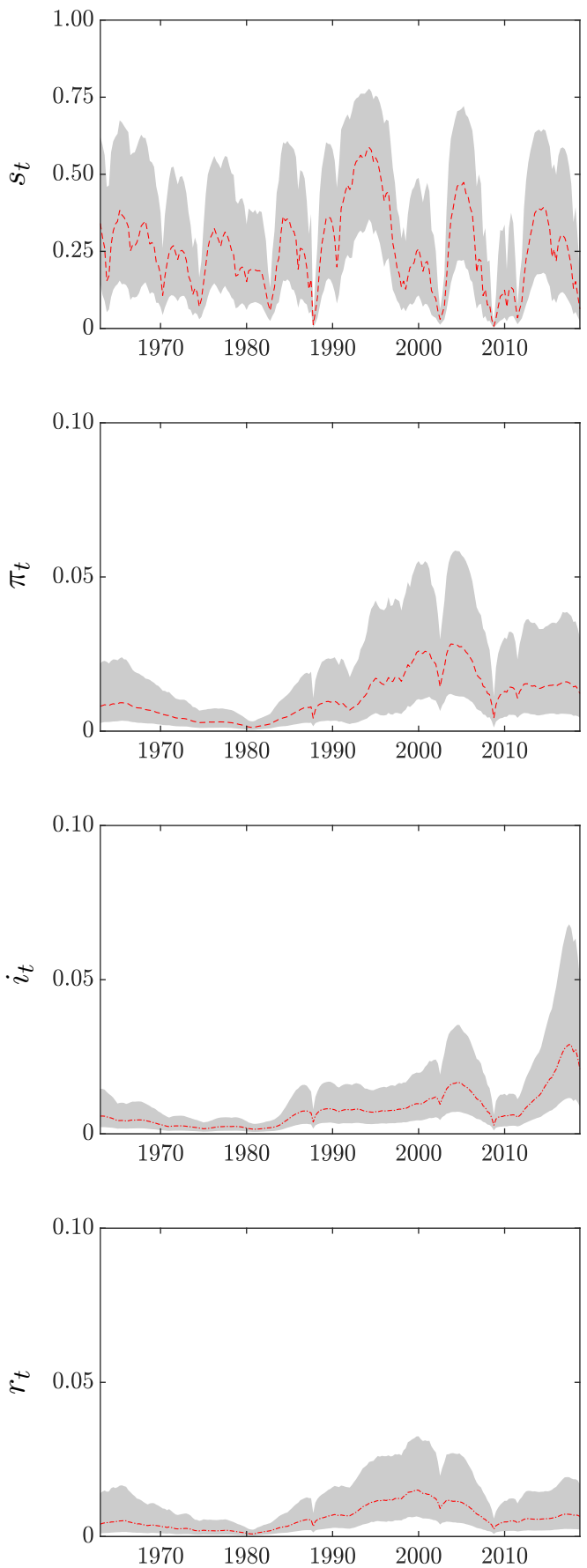

2-year horizon
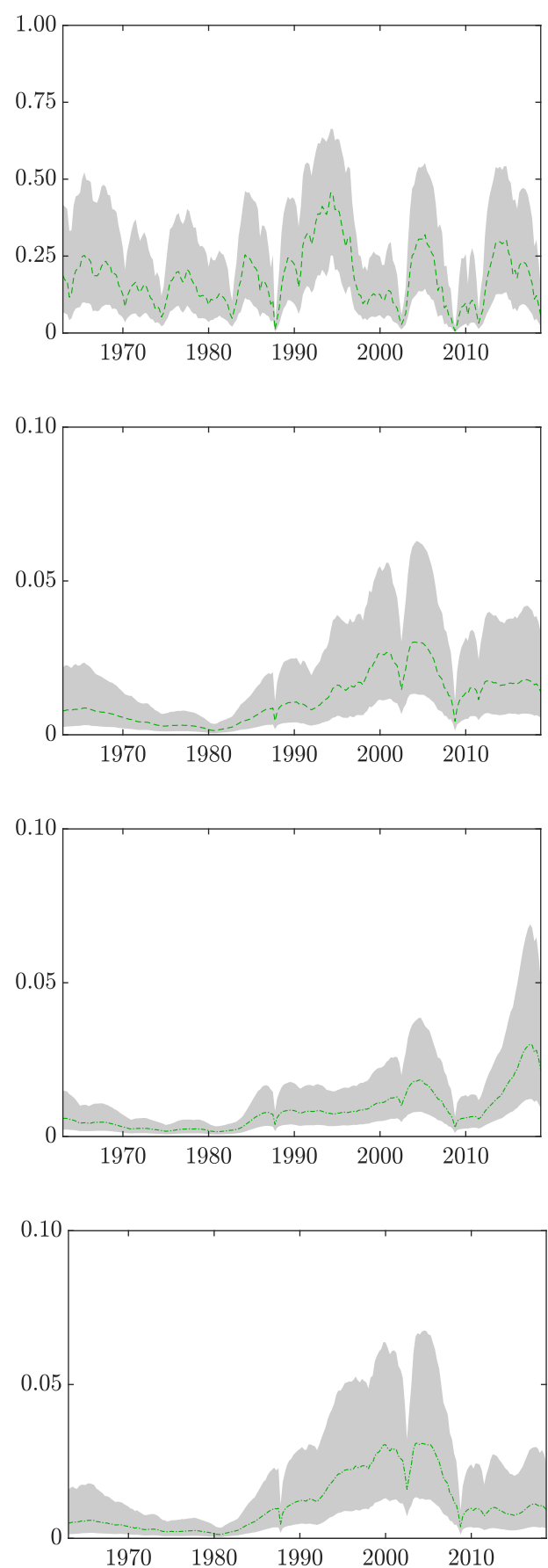

Note: FEVDs at 1-year and 2-year horizons. Order of the VAR: $\mathbf{y}_{t}=\left[S \& P 100_{t}, s_{t}, \pi_{t}, i_{t}, r_{t}\right]$.

paths are mostly indistinguishable. Although we have shown earlier that the sensitivity of macroeconomic variables to stock market volatility shocks (as evident from the impulse 
Figure 10: Actual and counterfactual paths of variables
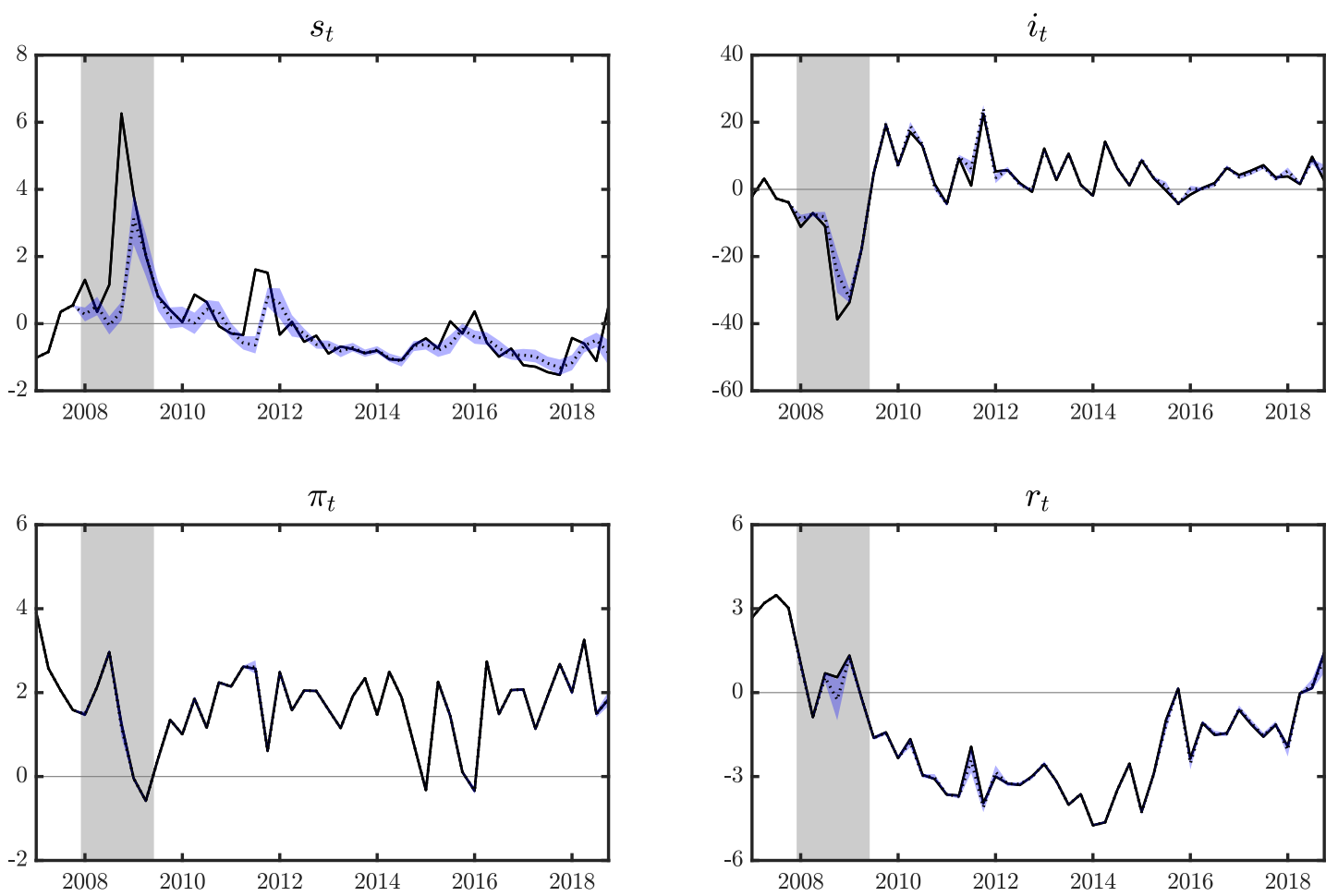

Note: Each panel compares the actual and counterfactual path of the variables. Black solid lines depict the actual path of the variables, and the dotted line is the posterior median. Blue shaded regions represent the posterior $68 \%$ credible interval, while the vertical gray-shaded region in the background depicts NBER recessions.

responses) has increased since the financial crisis, the effect of muting these shocks turn out to be inconsequential, except for 2008Q4. Therefore, it appears that financial uncertainty shocks played a minor role in the U.S. during and after the financial crisis.

\section{Conclusion}

What are the time-varying effects of financial uncertainty shocks on investment dynamics? We answer this question by estimating a time-varying parameter VAR with stochastic volatility using post-WWII U.S. data. The main findings of the paper can be summarized as follows.

First, we show that the predictability and persistence of uncertainty vary over time and are negatively correlated with uncertainty shock volatility, implying that uncertainty predictability and persistence rise after recessions. Second, we confirm that financial 
uncertainty shocks are contractionary shocks that result in statistically significant decline in investment growth. However, the "volatility overshoot" effect of uncertainty shock, consistent with the "wait-and-see" behavior of investment, is only evident since the 1990s. Such effect is found to coincide with a larger decline in interest rates, suggesting the rebound effect of investment may be partly driven by endogenously expansionary monetary policy. Third, we find the comovement between investment growth and inflation following uncertainty shocks to be negative in the period between the 1960s and the mid-1980s while turning positive since the 2000s. Hence, uncertainty shocks do not always act as aggregate "demand shocks". Fourth, the impact of uncertainty shock on investment growth has declined over time, which is consistent with a stronger response of interest rate to inflation. We also find the impact of such shocks to be more pronounced when the zero lower bound binds. Finally, despite the statistically significant impact of uncertainty shocks on investment and other macroeconomic variables, we find these shocks to play only a marginal role in explaining macroeconomic fluctuations over time, including in the Great Recession period. Overall, our results provide limited evidence to the notion that financial uncertainty shocks play an important role in driving aggregate fluctuations. 


\section{References}

Alexopoulos, M. and Cohen, J. (2009), Uncertain Times, Uncertain Measures, Working paper 352, Department of Economics, University of Toronto.

Bachmann, R., Elstner, S., and Sims, E.R. (2013), "Uncertainty and Economic Activity: Evidence from Business Survey Data", American Economic Journal: Macroeconomics 5 (2), pp. 217-249.

Baker, S.R., Bloom, N., and Davis, S.J. (2016), "Measuring Economic Policy Uncertainty", Quarterly Journal of Economics 131 (4), pp. 1593-1636.

Basu, S. and Bundick, B. (2017), "Uncertainty Shocks in a Model of Effective Demand", Econometrica 85 (3), pp. 937-958.

Benati, L. (2016), Economic Policy Uncertainty, the Great Recession, and the Great Depression, mimeo.

Benati, L. and Surico, P. (2008), "Evolving U.S. Monetary Policy and the Decline of Inflation Predictability", Journal of the European Economic Association 6 (2/3), pp. 634646.

Bloom, N. (2009), "The Impact of Uncertainty Shocks", Econometrica 77 (3), pp. 623-685.

Bloom, N., Bond, S., and Van Reenen, J. (2007), "Uncertainty and Investment Dynamics", Review of Economic Studies 74 (2), pp. 391-415.

Bloom, N., Floetotto, M., Jaimovich, N., Saporta-Eksten, I., and Terry, S.J. (2018), "Really Uncertain Business Cycles", Econometrica 86 (3), pp. 1031-1065.

Caggiano, G., Castelnuovo, E., and Groshenny, N. (2014), "Uncertainty Shocks and Unemployment Dynamics in U.S. Recessions", Journal of Monetary Economics 67, pp. 7892.

Caggiano, G., Castelnuovo, E., and Pellegrino, G. (2017), "Estimating the Real Effects of Uncertainty Shocks at the Zero Lower Bound", European Economic Review 100, pp. $257-272$.

Caldara, D., Fuentes-Albero, C., Gilchrist, S., and Zakrajšek, E. (2016), "The Macroeconomic Impact of Financial and Uncertainty Shocks", European Economic Review 88, pp. $185-207$. 
Castelnuovo, E. (2019), Domestic and Global Uncertainty: A Survey and Some New Results, Working paper 75/2019, Centre for Applied Macroeconomic Analysis.

Castelnuovo, E. and Tran, T.D. (2017), "Google it Up! A Google Trends-based Uncertainty Index for the United States and Australia", Economics Letters 161, pp. 149-153.

Christiano, L.J., Motto, R., and Rostagno, M. (2014), "Risk Shocks", American Economic Review 104 (1), pp. 27-65.

Clarida, R., Gali, J., and Gertler, M. (2000), "Monetary Policy Rules and Macroeconomic Stability: Evidence and Some Theory", Quarterly Journal of Economics 115 (1), pp. $147-180$.

Cogley, T., Primiceri, G.E., and Sargent, T.J. (2010), "Inflation-Gap Persistence in the US", American Economic Journal: Macroeconomics 2 (1), pp. 43-69.

Cogley, T. and Sargent, T.J. (2001), "Evolving Post-World War II U.S. Inflation Dynamics", NBER Macroeconomics Annual 2001, ed. by B.S. Bernanke and K. Rogoff, vol. 16, Cambridge, MA: MIT Press, pp. 331-388.

- (2005), "Drift and Volatilities: Monetary Policies and Outcomes in the Post WWII US", Review of Economic Dynamics 8 (2), pp. 262-302.

Davig, T. and Leeper, E.M. (2007), "Generalizing the Taylor Principle", American Economic Review 97 (3), pp. 607-635.

de Jong, P. and Shephard, N. (1995), "The Simulation Smoother for Time Series Models", Biometrica 82 (2), pp. 339-350.

Fasani, S. and Rossi, L. (2018), "Are Uncertainty Shocks Aggregate Demand Shocks?", Economics Letters 167, pp. 142-146.

Ferníndez-Villaverde, J., Guerrón-Quintana, P., Kuester, K., and Rubio-Ramírez, J. (2015), "Fiscal Volatility Shocks and Economic Activity", American Economic Review 105 (11), pp. 3352-3384.

Fernández-Villaverde, J., Guerrón-Quintana, P., Rubio-Ramírez, J.F., and Uribe, M. (2011), "Risk Matters: The Real Effects of Volatility Shocks", American Economic Review 101 (6), pp. 2530-61. 
Fernández-Villaverde, J. and Rubio-Ramírez, J.F. (2008), "How Structural Are Structural Parameters?", NBER Macroeconomics Annual 200\%, ed. by D. Acemoglu, K. Rogoff, and M. Woodford, vol. 22, Chicago, Illinois: University of Chicago Press, pp. 83-137.

Gilchrist, S., Sim, J.W., and Zakrajšek, E. (2014), Uncertainty, Financial Frictions, and Investment Dynamics, Working paper 20038, NBER.

Görtz, C., Tsoukalas, J., and Zanetti, F. (2016), News Shocks Under Financial Frictions, Working paper 2016-15, Business School - Economics, University of Glasgow.

Greasley, D. and Madsen, J.B. (2006), "Investment and Uncertainty: Precipitating the Great Depression in the United States", Economica 73 (291), pp. 393-412.

Haque, Q. (2019), Monetary Policy, Inflation Target and the Great Moderation: An Empirical Investigation, Working paper 44/2019, Centre for Applied Macroeconomic Analysis.

Haque, Q., Groshenny, N., and Weder, M. (2018), Do We Really Know that U.S. Monetary Policy Was Destabilizing in the 1970s?, Working paper 23/2018, Centre for Applied Macroeconomic Analysis.

Istrefi, K. and Mouabbi, S. (2018), "Subjective Interest Rate Uncertainty and the Macroeconomy: A Cross-country Analysis", Journal of International Money and Finance 88, pp. 296-313.

Johannsen, B.K. (2014), When Are the Effects of Fiscal Policy Uncertainty Large?, Working paper 2014-40, Financial and Economics Discussion Series, Board of Governors of the Federal Reserve System.

Jurado, K., Ludvigson, S.C., and Ng, S. (2015), "Measuring Uncertainty", American Economic Review 105 (3), pp. 1177-1216.

Justiniano, A., Primiceri, G.E., and Tambalotti, A. (2010), "Investment Shocks and Business Cycles", Journal of Monetary Economics 57 (2), pp. 132-145.

Katayama, M. and Kim, K.H. (2018), "Uncertainty Shocks and the Relative Price of Investment Goods", Review of Economic Dynamics 30, pp. 163-178.

Leahy, J.V. and Whited, T.M. (1996), "The Effect of Uncertainty on Investment: Some Stylized Facts", Journal of Money, Credit and Banking 28 (1), pp. 64-83. 
Leduc, S. and Liu, Z. (2016), "Uncertainty Shocks are Aggregate Demand Shocks", Journal of Monetary Economics 82, pp. 20-35.

Liu, P., Theodoridis, K., Mumtaz, H., and Zanetti, F. (2019), "Changing Macroeconomic Dynamics at the Zero Lower Bound", Journal of Business 83 Economic Statistics 37 (3), pp. 391-404.

Lubik, T.A. and Schorfheide, F. (2004), "Testing for Indeterminacy: An Application to US Monetary Policy", American Economic Review 94 (1), pp. 190-217.

Ludvigson, S.C., Ma, S., and Ng, S. (2018), Uncertainty and Business Cycles: Exogenous Impulse or Endogenous Response?, Working paper 21803, NBER.

Mumtaz, H. and Theodoridis, K. (2018), "The Changing Transmission of Uncertainty Shocks in the U.S.", Journal of Business \& Economic Statistics 36 (2), pp. 239-252.

Mumtaz, H. and Zanetti, F. (2013), "The Impact of the Volatility of Monetary Policy Shocks", Journal of Money, Credit and Banking 45 (4), pp. 535-558.

Nakajima, J. (2011), "Time-Varying Parameter VAR Model with Stochastic Volatility: An Overview of Methodology and Empirical Applications", Monetary and Economic Studies 29, pp. 107-142.

Primiceri, G.E. (2005), "Time Varying Structural Vector Autoregressions and Monetary Policy", Review of Economic Studies 72 (3), pp. 821-852.

Romer, C.D. (1990), "The Great Crash and the Onset of the Great Depression", Quarterly Journal of Economics 105 (3), pp. 597-624.

Rossi, B. and Sekhposyan, T. (2015), "Macroeconomic Uncertainty Indices Based on Nowcast and Forecast Error Distributions", American Economic Review 105 (5), pp. 65055.

Scotti, C. (2016), "Surprise and Uncertainty Indexes: Real-time Aggregation of Realactivity Macro-surprises", Journal of Monetary Economics 82, pp. 1-19.

Shephard, N. and Pitts, M. (1997), "Likelihood Analysis of Non-Gaussian Measurement Time Series", Biometrica 84 (3), pp. 653-667. 
Sims, C.A. (2001), "Comment on Sargent and Cogley's 'Evolving Post-World War II U.S. Inflation Dynamics"”, NBER Macroeconomics Annual 2001, ed. by B.S. Bernanke and K. Rogoff, vol. 16, Cambridge, MA: MIT Press, pp. 373-379.

Smets, F. and Wouters, R. (2007), "Shocks and Frictions in US Business Cycles: A Bayesian DSGE Approach", American Economic Review 97 (3), pp. 586-606.

Watanabe, T. and Omori, Y. (2004), "A Multi-move Sampler for Estimating Non-Gaussian Time Series Models: Comments on Shephard and Pitt (1997)", Biometrica 91 (1), pp. 246-248.

Wu, J.C. and Xia, F.D. (2016), "Measuring the Macroeconomic Impact of Monetary Policy at the Zero Lower Bound", Journal of Money, Credit and Banking 48 (2-3), pp. 253291. 


\title{
Supplement to "Empirical evidence on the dynamics of investment under uncertainty in the U.S."
}

\author{
Qazi Haque* \\ Leandro M. Magnusson ${ }^{\dagger}$ \\ The University of Western Australia \\ The University of Western Australia \\ Centre for Applied Macroeconomic Analysis \\ Kazuki Tomioka ${ }^{\ddagger}$ \\ University of Rochester
}

November 20, 2019

\section{Contents}

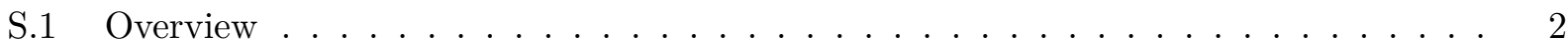

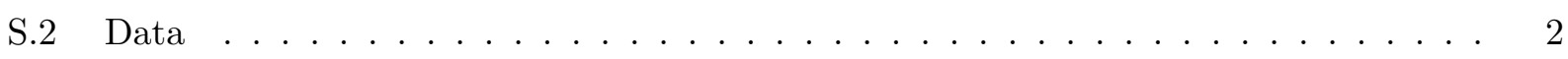

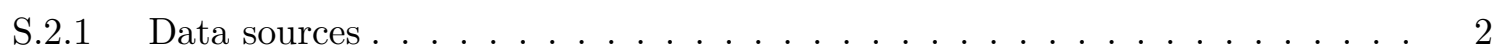

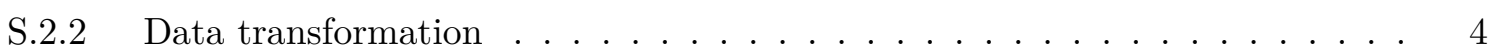

S.3 Markov chain Monte Carlo method for sampling the VAR parameters . . . . . . . 5

S.3.1 Sampling coefficient $\left(\mathbf{b}^{T}\right) \ldots \ldots \ldots \ldots \ldots$

S.3.2 Sampling simultaneous relation $\left(\mathbf{a}^{T}\right) \ldots \ldots \ldots 6$

S.3.3 Sampling volatility $\left(\mathbf{h}^{T}\right) \ldots \ldots \ldots \ldots \ldots$

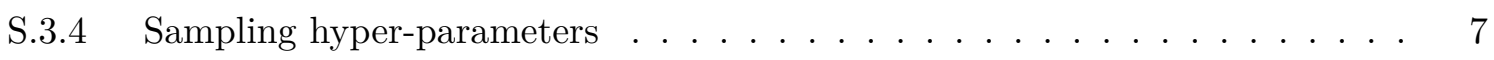

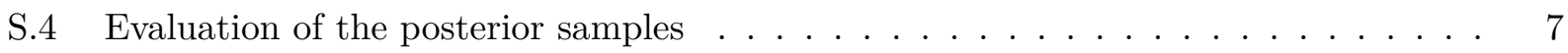

S.5 Computation of various statistics . . . . . . . . . . . . . 7

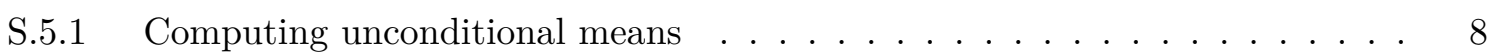

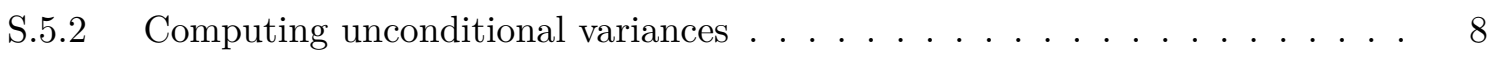

S.5.3 Computing impulse response functions . . . . . . . . . . . . . . . . . 10

S.5.4 Computing predictability and persistence . . . . . . . . . . 11

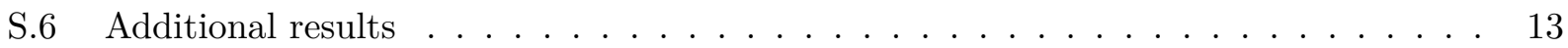

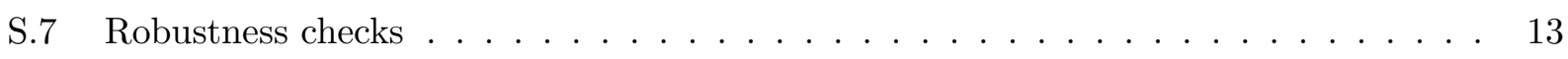

S.7.1 Alternative proxy for uncertainty . . . . . . . . . . . . . 14

${ }^{*}$ CAMA and Department of Economics, Business School, The University of Western Australia. M251, 35 Stirling Hwy, Crawley WA 6009, Australia. Email: qazi.haque@uwa.edu.au.

${ }^{\dagger}$ Department of Economics, Business School, The University of Western Australia. M251, 35 Stirling Hwy, Crawley WA 6009, Australia. Email: leandro.magnusson@uwa.edu.au. (corresponding author)

${ }^{\ddagger}$ Department of Economics, Harkness Hall, University of Rochester, Rochester, NY 14627. Email: ktomioka@ur.rochester.edu. 
S.7.2 Prior sensitivity . . . . . . . . . . . . . . . . . . . 15

S.7.3 Different ordering . . . . . . . . . . . . . . . . . 15

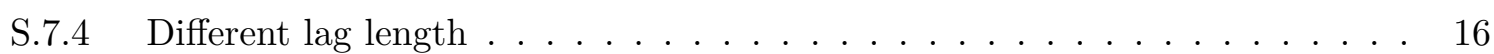

\section{S.1 Overview}

This Supplement details the data, and the econometric estimation methods employed in the paper "Empirical evidence on the dynamics of investment under uncertainty in the U.S.", and also provides some additional results. Sources of the data and corresponding transformations are offered in § S.2. Technical details of the Markov chain Monte Carlo (MCMC) method employed to estimate time varying VAR are offered in $\S$ S.3. Posterior evaluation of the model and the statistics computed from it are offered in $\S \mathrm{S} .4$ and $\S \mathrm{S} .5$ respectively. Additional results from the manuscript and further robustness checks are provided in $\S \mathrm{S} .6$ and $\S \mathrm{S} .7$ respectively.

Throughout the text, we use the following notation: Bold lowercase alphabet and Greek symbols (e.g. a and $\boldsymbol{\omega}$ ) denote vectors, while uppercase counterparts (e.g. $\mathbf{A}$ and $\boldsymbol{\Omega}$ ) denote matrices. Of particular note are matrices $\mathbf{I}_{n}$ and $\mathbf{O}_{n}$. They respectively denote the identity and the null matrix of dimension $n$. Unless otherwise stated, all other notations we use are relatively standard in the literature.

\section{S.2 Data}

\section{S.2.1 Data sources}

\section{Uncertainty proxy}

VXO: The data for this proxy is sourced from the Chicago Board of Options Exchange (CBOE) and retrieved from FRED, https://fred.stlouisfed.org/series/VXOCLS. This index is unavailable before 1986. Following Bloom (2009), pre-1986 monthly return volatilities are computed by employing the monthly standard deviation of the daily S\&P500 index normalized to the same mean and variance as the VXO index when they overlap from 1986 onward.

GSZ: This proxy is introduced in Gilchrist et al. (2014) and retreived from the Online Appendix of Caldara et al. (2016).

JLN: The financial uncertainty measure proposed in Jurado et al. (2015), retreived from Sydney C Ludvigson's site https://www.sydneyludvigson. com/data-and-appendixes/. 


\section{Investment and real GDP}

In the benchmark analysis, the measure of investment follows that of Justiniano et al. (2010) and is the sum of personal consumption expenditure on durable goods and gross private domestic investment. In the robustness section, we use fixed private investment following Smets and Wouters (2007). Additionally, we also estimate the model using real gross domestic product as a measure of economic activity instead of investment.

\section{Gross Private Domestic Investment [GPDI]}

Billions of Dollars, Seasonally Adjusted Annual Rate; Source: U.S. Bureau of Economic Analysis; FRED. https://fred.stlouisfed.org/series/GPDI

Fixed Private Investment [FPI]

Billions of Dollars, Seasonally Adjusted Annual Rate; Source: U.S. Bureau of Economic Analysis; FRED. https://fred.stlouisfed.org/series/FPI

Personal Consumption Expenditures: Durable Goods [PCDG]

Billions of Dollars, Seasonally Adjusted Annual Rate; Source: U.S. Bureau of Economic Analysis; FRED. https://fred.stlouisfed.org/series/PCDG

\section{Real Gross Domestic Product Per Capita [A939RX0Q048SBEA]}

Billions of Chained 2012 Dollars, Seasonally Adjusted Annual Rate; Source: U.S. Bureau of Economic Analysis; FRED. https://fred.stlouisfed.org/series/A939RX0Q048SBEA

\section{Prices and interest rates}

\section{Gross Domestic Product: Implicit Price Deflator [GDPDEF]}

Index 2012=100, Seasonally Adjusted; Source: U.S. Bureau of Economic Analysis; FRED. https://fred.stlouisfed.org/series/GDPDEF.

\section{Gross Private Domestic Investment: Implicit Price Deflator [A006RD3Q086SBEA]} Index 2012=100, Seasonally Adjusted; Source: U.S. Bureau of Economic Analysis; FRED. https://fred.stlouisfed.org/series/A006RD3Q086SBEA.

Gross Private Domestic Investment: Fixed Investment: Implicit Price Deflator [A007RD3Q086SBEA]

Index 2012=100, Seasonally Adjusted; Source: U.S. Bureau of Economic Analysis; FRED. https://fred.stlouisfed.org/series/A007RD3Q086SBEA\#0.

Personal Consumption Expenditures: Durable goods: Implicit Price Deflator [DDURRD3Q086SBEA]

Index 2012=100, Seasonally Adjusted; Source: U.S. Bureau of Economic Analysis; FRED. https://fred.stlouisfed.org/series/DDURRD3Q086SBEA. 


\section{Effective Federal Funds Rate [FEDFUNDS]}

Percent, Not Seasonally Adjusted; Source: Board of Governors of the Federal Reserve System; FRED. https://fred.stlouisfed.org/series/FEDFUNDS.

\section{Wu-Xia Shadow Federal Funds Rate}

Source: Wu and Xia (2016); retrieved from Federal Reserve Bank of Atlanta. https://www . frbatlanta.org/cqer/research/shadow_rate.aspx?panel=1

Note: When the shadow fed funds rate is at least 25 basis points, it equals the federal funds rate. At the zero lower bound, the shadow rate uses information from the entire yield curve to summarize the stance of monetary policy.

\section{Moody's Seasoned Baa Corporate Bond Yield [BAA]}

Percent, Not Seasonally Adjusted; Source: Moody's; FRED. https://fred.stlouisfed. org/series/BAA\#0.

\section{0-Year Treasury Constant Maturity Rate [DGS30]}

Percent, Not Seasonally Adjusted; Source: Board of Governors of the Federal Reserve System; FRED. https://fred.stlouisfed.org/series/DGS30\#0.

\section{0-Year Treasury Constant Maturity Rate [GS20]}

Percent, Not Seasonally Adjusted; Source: Board of Governors of the Federal Reserve System; FRED. https://fred.stlouisfed.org/series/GS20\#0.

\section{Other variables}

\section{Real S\&P100 Composite Price}

Source: Robert Shiller's personal website. http://www.econ.yale.edu/ shiller/data.htm.

\section{Consumer Sentiment}

Source: University of Michigan. https://data.sca.isr.umich.edu/data-archive/mine. php.

Note: This index is calculated as an average of expectations about business conditions over the next year, expectations about aggregate business conditions over the next five years and expectations about personal financial conditions over the next year.

\section{S.2.2 Data transformation}

In this subsection, we first outline the way in which the data were transformed for the analyses in the manuscript.

Uncertainty: Quarterly average of the monthly series, demeaned and standardized.

Inflation: Log difference of the quarterly implicit GDP price deflator, multiplied by 400 to convert it into annualized rate. 
Investment: Investment series is first divided by the civilian non-institutional population (16 years or over) to convert into per capita terms and the resulting per capita series is then deflated into 2012 Dollars using the respective implicit price deflators. Annualized growth rate is computed by taking the log difference of the resulting series and multiplying by 400 .

Real interest rate: Ex-ante real interest rate is proxied via the ex-post real interest rate, by taking the difference between the Wu and Xia (2016) Shadow Federal Funds Rate and the period ahead realized inflation rate, which is in turn calculated using the GDP deflator.

Credit Spread: Difference between the Baa 30-year yield and the 30-year Treasury bond yield. In periods when the 30-year bond is missing we use the 20-year treasury bond yield as in Bachmann et al. (2013).

S\&P100: Detrended by applying the Hodrick-Prescott filter to the log of the S\&P100 index with a smoothing parameter of 1600 .

Consumer sentiment: Demeaned and standardized the quarterly series.

\section{S.3 Markov chain Monte Carlo method for sampling the VAR pa- rameters}

In order to analyze the evolving dynamics of investment under uncertainty, the paper employs a time-varying VAR where all model coefficients and covariances drift with time. The model has become standard in the macroeconometrics literature and a variety of Bayesian Markov chain Monte Carlo (MCMC) estimation methods have been proposed. Earlier applications of the model to U.S. and Japanese macroeconomic data are in Cogley and Sargent (2005), Primiceri (2005), Nakajima (2011), and Nakajima et al. (2011) and they offer detailed posterior computation methods. Hence, in what follows, we only outline the MCMC procedures used in the paper and refer the readers to the references provided for a more formal treatment of the individual sampling algorithms.

This paper augments the MCMC routine developed by Nakajima $(2011)^{1}$ to draw samples from the joint posterior density function, $p\left(\mathbf{b}^{T}, \mathbf{a}^{T}, \mathbf{h}^{T}, \mathbf{Q}, \mathbf{S}, \mathbf{W} \mid \mathbf{y}^{T}\right)$. This is achieved by the following MCMC routine that cycles through:

1. Initialize $\mathbf{b}^{T}, \mathbf{a}^{T}, \mathbf{h}^{T}, \mathbf{Q}, \mathbf{S}, \mathbf{W}$

2. Sample $\mathbf{b}^{T}$ from $p\left(\mathbf{b}^{T} \mid \mathbf{a}^{T}, \mathbf{h}^{T}, \mathbf{Q}, \mathbf{y}^{T}\right)$

3. Sample $\mathbf{Q}$ from $p\left(\mathbf{Q} \mid \mathbf{b}^{T}\right)$

4. Sample $\mathbf{a}^{T}$ from $p\left(\mathbf{a}^{T} \mid \mathbf{b}^{T}, \mathbf{h}^{T}, \mathbf{S}, \mathbf{y}^{T}\right)$

5. Sample $\mathbf{S}$ from $p\left(\mathbf{S} \mid \mathbf{a}^{T}\right)$

\footnotetext{
${ }^{1}$ The original MatLaB program written by Jouchi Nakajima is available on his personal website, https://sites . google.com/site/jnakajimaweb/.
} 
6. Sample $\mathbf{h}^{T}$ from $p\left(\mathbf{h}^{T} \mid \mathbf{b}^{T}, \mathbf{a}^{T}, \mathbf{W}, \mathbf{y}^{T}\right)$

7. Sample $\mathbf{W}$ from $p\left(\mathbf{W} \mid \mathbf{h}^{T}\right)$

8. Compute $\operatorname{det}\left(\mathbf{M}_{t}-\boldsymbol{\lambda}_{t} \mathbf{I}_{n P}\right)=0$ for $\boldsymbol{\lambda}_{t}$ :

if $\max \left(\left|\boldsymbol{\lambda}_{t}\right|\right) \geq 1$, reject the draw

else $\max \left(\left|\boldsymbol{\lambda}_{t}\right|\right)<1$ store for posterior analyses

9. Back to 2

Note that $p(\bullet \mid \bullet)$ denotes conditional density functions, and recall that $\mathbf{b}^{T}=\left\{\boldsymbol{\beta}_{t}\right\}_{t=P+1}^{T}, \mathbf{a}^{T}=$ $\left\{\boldsymbol{\alpha}_{t}\right\}_{t=P+1}^{T}, \mathbf{h}^{T}=\left\{\log \boldsymbol{\sigma}_{t}^{2}\right\}_{t=P+1}^{T}$ and $\mathbf{y}^{T}=\left\{\mathbf{y}_{t}\right\}_{t=P+1}^{T}$. Steps 2 and 4 are executed using the simulation smoother, while step 6 is executed using the block sampler. Steps 3, 5, and 7 are conducted by generating samples from the inverse Gamma distribution.

\section{S.3.1 Sampling coefficient $\left(b^{T}\right)$}

The time varying VAR coefficient, $\mathbf{b}^{T}$, is sampled from the conditional posterior density, $p\left(\mathbf{b}^{T} \mid \mathbf{a}^{T}, \mathbf{h}^{T}, \mathbf{Q}, \mathbf{y}^{T}\right)$, with a prior on the initial state $\boldsymbol{\beta}_{P+1} \sim \mathbf{N}(\mathbf{0}, 10 \mathbf{I})$, where $\mathbf{I}$ is an identity matrix of the size $\operatorname{dim}\left(\boldsymbol{\beta}_{t}\right)$. The joint sampling of $\mathbf{b}^{T}$ conditioned on rest of the parameters is much more efficient than to sample $\boldsymbol{\beta}_{\tau}$ one at a time conditioned on $\mathbf{a}^{T}, \mathbf{h}^{T}, \mathbf{Q}, \mathbf{y}^{T}$ but also on $\boldsymbol{\beta}_{t}$ for $\tau \neq t$. This is achieved by using the simulation smoother developed by de Jong and Shephard (1995).

\section{S.3.2 Sampling simultaneous relation $\left(\mathrm{a}^{T}\right)$}

Consistent with the sampling of $\mathbf{b}^{T}$, the time varying simultaneous relation, $\mathbf{a}^{T}$, is also sampled from the conditional posterior distribution, $p\left(\mathbf{a}^{T} \mid \mathbf{b}^{T}, \mathbf{h}^{T}, \mathbf{S}, \mathbf{y}^{T}\right)$ using the simulation smoother of de Jong and Shephard (1995) with $\boldsymbol{\alpha}_{P+1} \sim \mathbf{N}(\mathbf{0}, 10 \mathbf{I})$, where $\mathbf{I}$ is an identity matrix of the size $\operatorname{dim}\left(\boldsymbol{\alpha}_{t}\right)$.

\section{S.3.3 Sampling volatility $\left(\mathbf{h}^{T}\right)$}

Stochastic volatility, $\mathbf{h}^{T}$, is sampled using the block sampler of Shephard and Pitts (1997), corrected by Watanabe and Omori (2004), which samples $\mathbf{h}^{T}$ directly from the exact conditional posterior density, $p\left(\mathbf{h}^{T} \mid \mathbf{b}^{T}, \mathbf{a}^{T}, \mathbf{W}, \mathbf{y}^{T}\right)$. We place a prior $\log \boldsymbol{\sigma}_{P+1}^{2} \sim \mathbf{N}(\mathbf{0}, 10 \mathbf{I})$ as the initial state, where $\mathbf{I}$ is an identity matrix of the $\operatorname{size} \operatorname{dim}\left(\boldsymbol{\sigma}_{t}^{2}\right)$. Note that the algorithm employed here departs from, perhaps the more popular mixture sampler of Kim et al. (1998), used by Primiceri (2005). The mixture sampler samples log volatility from the density function that approximates the posterior conditional distribution, while the appeal of the block sampler is its sampling from the exact conditional posterior. 


\section{S.3.4 Sampling hyper-parameters}

The hyper-parameters on the time varying VAR can be drawn from their respective distribution conditional on their respective parameters. Our model takes the form where the hyper-parameters $\mathbf{Q}, \mathbf{S}$ and $\mathbf{W}$ are diagonal. These assumptions are typically placed for convenience to simplify the estimation method and to economize on the estimation of parameters. The removal of cross-equation restrictions via diagonalization are reported to have minimal effect on the posterior estimates of the parameters (Primiceri, 2005; Nakajima, 2011).

The conditional posterior distribution of the diagonal elements $\forall F_{i}=Q_{i}, S_{i}, W_{i}$ given $\boldsymbol{\delta}^{T}=$ $\mathbf{b}^{T}, \mathbf{a}^{T}, \mathbf{h}^{T}$ is

$$
p\left(\iota_{i} \mid \boldsymbol{\delta}^{T}\right) \sim \mathrm{G}^{-1}\left(\frac{\widehat{v}_{\mathfrak{\delta}^{T}}}{2}, \frac{\widehat{V}_{\iota, i}}{2}\right),
$$

where $\mathrm{G}^{-1}$ denotes the inverse-Gamma distribution; $\widehat{v}_{\mathfrak{\delta}^{T}}=v_{\mathfrak{\delta}^{T}, 0}+T-P-1$; and $\widehat{V}_{F, i}=V_{F, 0}+$ $\sum_{t=P+1}^{T-1}\left(F_{i, t+1}-F_{i, t}\right)^{2}$. Notice that $v_{\mathfrak{\delta}^{T}, 0}$ and $V_{F, 0}$ are the arguments for the initial states of the parameters, which in this paper is set as $F_{i} \sim \mathrm{G}^{-1}(40 / 2,0.004 / 2)$ for $F_{i}=Q_{i}$ and $F_{i} \sim \mathrm{G}^{-1}(12 / 2,0.01 / 2)$ for the rest.

Note that the diagonal assumption on $\mathbf{W}$ and the individual $\log \sigma_{i, t}^{2}$ together define traditional univariate stochastic volatility model (Jacquier et al., 1994) except we place a geometric random walk assumption rather than the stationary $\mathrm{AR}(1)$ process to capture of non-stationary behaviors. This aids to economize on the estimation burden that hinders high dimensional model like the time-varying VAR.

\section{S.4 Evaluation of the posterior samples}

Figure S.1 plots the sample traces of MCMC draws, their respective posterior distributions and the autocorrelation functions for selected parameters in the time-varying VAR model. Note that $\widehat{\iota}_{1,50}$ denotes the first element of the parameters at $t=50$, and $\sqrt{Q}_{1}, \sqrt{S}_{1}, \sqrt{W}_{1}$ all denote the square roots of the $(1,1)$ elements in the respective matrices. We observe that the autocorrelation functions decay quickly and that their sample traces are stable, providing evidence for mixing of the chains.

[Figure S.1 here]

\section{S.5 Computation of various statistics}

In this section, we describe in detail the statistics computed in the main text. Most of the statistics are time varying counterpart of what is described in detail in standard time series econometrics textbooks such as Hamilton (1994) and Lütkepohl (2005). Some are from academic sources and are cited accordingly. 


\section{S.5.1 Computing unconditional means}

Consider equation (1) of the main text reprinted here

$$
\mathbf{y}_{t}=\mathbf{c}_{t}+\sum_{p=1}^{P} \mathbf{B}_{p, t} \mathbf{y}_{t-p}+\mathbf{u}_{t}, \quad \mathbf{u}_{t} \sim \mathrm{N}\left(\mathbf{0}, \boldsymbol{\Omega}_{t}\right)
$$

where $\mathbf{y}_{t}$ is an $n \times 1$ vector of endogenous variables, $\mathbf{c}_{t}$ is an $n \times 1$ column vector of intercepts, $\mathbf{B}_{p, t}$ is an $n \times n$ matrix containing the $p^{t h}$ lag autoregressive coefficients, and $\mathbf{u}_{t}$ are heteroscedastic unobservable shocks with variance-covariance matrix $\boldsymbol{\Omega}_{t}$. This can be rewritten in companion form

$$
\mathbf{z}_{t}=\mathbf{v}_{t}+\mathbf{M}_{t} \mathbf{z}_{t-1}+\mathbf{e}_{t}
$$

which, with $P=2$ is

$$
\left[\begin{array}{c}
\mathbf{y}_{t} \\
\mathbf{y}_{t-1}
\end{array}\right]=\left[\begin{array}{c}
\mathbf{c}_{t} \\
\mathbf{0}_{n, t}
\end{array}\right]+\left[\begin{array}{cc}
\mathbf{B}_{1, t} & \mathbf{B}_{2, t} \\
\mathbf{I}_{n, t} & \mathbf{O}_{n, t}
\end{array}\right]\left[\begin{array}{c}
\mathbf{y}_{t-1} \\
\mathbf{y}_{t-2}
\end{array}\right]+\left[\begin{array}{c}
\mathbf{u}_{t} \\
\mathbf{0}_{n, t}
\end{array}\right] .
$$

Beveridge and Nelson (1981) defines the stochastic trend in $\mathbf{z}_{t}$ as the value to which the series is expected to converge in the long run

$$
\boldsymbol{\mu}_{t}=\lim _{h \rightarrow \infty} \mathbb{E}_{t}\left(\mathbf{z}_{t+h}\right),
$$

which we approximate via the unconditional mean

$$
\boldsymbol{\mu}_{t} \approx\left(\mathbf{I}_{n P}-\mathbf{M}_{t}\right)^{-1} \boldsymbol{v}_{t}
$$

following the trend inflation literature (see, for e.g. Cogley and Sargent (2005) and Cogley et al. (2010)). Thus, elements of $\boldsymbol{\mu}_{t}$ are often interpreted as the 'core' or 'trend' of a variable.

\section{S.5.2 Computing unconditional variances}

The conditional mean, represented in companion form is

$$
\mathbf{v}_{t}=\left(\mathbf{I}_{n P}-\mathbf{M}_{t}\right) \boldsymbol{\mu}_{t}
$$

which when substituted it into (S.1) gives

$$
\mathbf{z}_{t}-\boldsymbol{\mu}_{t}=\mathbf{M}_{t}\left(\mathbf{z}_{t-1}-\boldsymbol{\mu}_{t}\right)+\mathbf{e}_{t},
$$

where, for notational convenience, denote $\widehat{\mathbf{z}}_{t}=\mathbf{z}_{t}-\boldsymbol{\mu}_{t}$, implying that the process $\widehat{\mathbf{z}}_{t}$ has zero mean. 
For a time invariant model, the unconditional variance-covariance matrix can be computed by

$$
\begin{aligned}
\underbrace{\widetilde{\boldsymbol{\Omega}}}_{n P \times n P} & =\mathbb{E}\left(\widehat{\mathbf{z}}_{t} \widehat{\mathbf{z}}_{t}^{\prime}\right), \\
& =\left[\begin{array}{llll}
\boldsymbol{\Gamma}(0) & \boldsymbol{\Gamma}(1) & \cdots & \boldsymbol{\Gamma}(P-1) \\
\boldsymbol{\Gamma}^{\prime}(1) & \boldsymbol{\Gamma}(0) & \cdots & \boldsymbol{\Gamma}(P-2) \\
\vdots & \vdots & \ddots & \vdots \\
\boldsymbol{\Gamma}^{\prime}(P-1) & \boldsymbol{\Gamma}^{\prime}(P-2) & \cdots & \boldsymbol{\Gamma}(0)
\end{array}\right],
\end{aligned}
$$

where each individual element $\boldsymbol{\Gamma}(h)$ denotes the autocovariance matrix that has the relationship

$$
\Gamma^{\prime}(h)=\Gamma(-h) .
$$

To compute the unconditional variance-covariance matrix that time varies, $\widetilde{\boldsymbol{\Omega}}_{t}$, we can proceed by substituting $\widehat{\mathbf{z}}_{t}=\mathbf{M}_{t}\left(\widehat{\mathbf{z}}_{t-1}\right)+\mathbf{e}_{t}$

$$
\begin{aligned}
\widetilde{\mathbf{\Omega}}_{t} & =\mathbb{E}_{t}\left[\left(\mathbf{M}_{t} \widehat{\mathbf{z}}_{t-1}+\mathbf{e}_{t}\right)\left(\mathbf{M}_{t} \widehat{\mathbf{z}}_{t-1}+\mathbf{e}_{t}\right)^{\prime}\right], \\
& =\mathbf{M}_{t} \mathbb{E}_{t}\left(\widehat{\mathbf{z}}_{t-1} \widehat{\mathbf{z}}_{t-1}^{\prime}\right) \mathbf{M}_{t}^{\prime}+\mathbb{E}_{t}\left(\mathbf{e}_{t} \mathbf{e}_{t}^{\prime}\right), \\
& =\mathbf{M}_{t} \widetilde{\mathbf{\Omega}}_{t-1} \mathbf{M}_{t}^{\prime}+\mathbf{V}_{t},
\end{aligned}
$$

where $\mathbf{V}_{t}$ is the companion form of the reduced form variance-covariance matrix. Applying the vec operator and the Kronecker product rule ${ }^{2}$ to both sides yields

$$
\operatorname{vec}\left(\widetilde{\boldsymbol{\Omega}}_{t}\right)=\left(\mathbf{M}_{t} \otimes \mathbf{M}_{t}\right) \operatorname{vec}\left(\widetilde{\boldsymbol{\Omega}}_{t-1}\right)+\operatorname{vec}\left(\mathbf{V}_{t}\right),
$$

hence

$$
\operatorname{vec}\left(\widetilde{\boldsymbol{\Omega}}_{t}\right)=\left(\mathbf{I}_{(n P)^{2}}-\mathbf{M}_{t} \otimes \mathbf{M}_{t}\right)^{-1} \operatorname{vec}\left(\mathbf{V}_{t}\right),
$$

with the approximation $\operatorname{vec}\left(\widetilde{\boldsymbol{\Omega}}_{t}\right) \approx \operatorname{vec}\left(\widetilde{\boldsymbol{\Omega}}_{t-1}\right)$. Thus, the unconditional variance-covariance matrix can be approximated by solving equation (S.3) for every time period. When $\operatorname{vec}\left(\widetilde{\boldsymbol{\Omega}}_{t}\right)$ is converted back into a matrix form, the first $n \times n$ elements are $\boldsymbol{\Gamma}_{t}(0)=\mathbf{K} \widetilde{\boldsymbol{\Omega}}_{t} \mathbf{K}^{\prime}$, where $\mathbf{K}=\left[\mathbf{I}_{n} \vdots \mathbf{0}_{n \times n(P-1)}\right]$ such that $\mathbf{K}^{\prime} \mathbf{K}=\mathbf{I}_{n P}$; and $\boldsymbol{\Gamma}_{t}(0)$ is the time varying unconditional covariance matrix. The diagonal elements characterize unconditional variance of variables, or put differently, the second moment counterpart of the Beveridge and Nelson (1981) trend.

An alternative method to approximate the unconditional covariance matrix is to follow Cogley and Sargent (2001) and use $h=120$ in the equation

$$
\boldsymbol{\Gamma}_{t}(0)=\sum_{h=0}^{120} \boldsymbol{\Phi}_{h, t} \boldsymbol{\Omega}_{t} \boldsymbol{\Phi}_{h, t}^{\prime} .
$$

For our dataset we find that there are no material differences in the results when either of the approximations are used.

\footnotetext{
${ }^{2}$ Let $\mathbf{A}, \mathbf{B}$, and $\mathbf{C}$ be matrices whose dimensions are such that the product $\mathbf{A B C}$ exists. Then, the Kronecker product rule vec $(\mathbf{A B C})=\left(\mathbf{C}^{\prime} \otimes \mathbf{A}\right) \operatorname{vec}(\mathbf{B})$ holds.
} 


\section{S.5.3 Computing impulse response functions}

Consider equation (2) of the main text reprinted here

$$
\mathbf{y}_{t}=\mathbf{X}_{t} \boldsymbol{\beta}_{t}+\mathbf{A}_{t}^{-1} \boldsymbol{\Sigma}_{t} \boldsymbol{\epsilon}_{t}, \quad t=P+1, \cdots, T,
$$

and note the decomposition of the reduced form residual term

$$
\mathbf{u}_{t}=\mathbf{A}_{t}^{-1} \boldsymbol{\Sigma}_{t} \boldsymbol{\epsilon}_{t}
$$

To achieve this decomposition in the companion form of the residual, $\mathbf{e}_{t}$, first define

$$
\mathbf{P}_{t}=\left[\begin{array}{c}
\mathbf{A}_{t}^{-1} \boldsymbol{\Sigma}_{t} \\
\mathbf{O}_{n}
\end{array}\right]
$$

such that the standard Cholesky factorization $\mathbf{P}_{t} \mathbf{P}_{t}^{\prime}=\mathbf{V}_{t}$ holds. Therefore, equation (S.1) can now be rewritten

$$
\mathbf{z}_{t}=\mathbf{v}_{t}+\mathbf{M}_{t} \mathbf{z}_{t-1}+\mathbf{P}_{t} \boldsymbol{\epsilon}_{t}
$$

Recall from $\S \mathrm{S} .3$, the rejection of unstable draws that produce an explosive root. The satisfaction of the stability condition allows the rewriting of the VAR into the vector moving-average, $\operatorname{VMA}(\infty)$ notation (a.k.a., Koyck transformation)

$$
\mathbf{y}_{t}=\mathbf{K} \mathbf{z}_{t}=\mathbf{K} \boldsymbol{\mu}_{t}+\mathbf{K} \sum_{h=0}^{\infty} \mathbf{M}_{t}^{h} \mathbf{e}_{t-h}
$$

where, as before, $\mathbf{K}=\left[\mathbf{I}_{n} \vdots \mathbf{0}_{n \times n(P-1)}\right]$ is a $(n \times n P)$ selection matrix with the property that $\mathbf{K}^{\prime} \mathbf{K}=\mathbf{I}_{n P}$. Thus the above equation admits the following representation

$$
\begin{aligned}
\mathbf{y}_{t} & =\mathbf{K} \boldsymbol{\mu}_{t}+\sum_{h=0}^{\infty} \mathbf{K} \mathbf{M}_{t}^{h} \mathbf{K}^{\prime} \mathbf{K} \mathbf{e}_{t-h}, \\
& =\mathbf{K} \boldsymbol{\mu}_{t}+\sum_{h=0}^{\infty} \mathbf{K} \mathbf{M}_{t}^{h} \mathbf{K}^{\prime} \mathbf{K}_{t} \mathbf{P}_{t} \boldsymbol{\epsilon}_{t-h}, \\
& =\mathbf{K} \boldsymbol{\mu}_{t}+\sum_{h=0}^{\infty} \boldsymbol{\Xi}_{t}(h) \boldsymbol{\epsilon}_{t-h}
\end{aligned}
$$

where $\boldsymbol{\Xi}_{t}(h)=\mathbf{K M}_{t}^{h} \mathbf{K}^{\prime} \mathbf{K} \mathbf{P}_{t}=\mathbf{K M}_{t}^{h} \mathbf{K}^{\prime} \mathbf{A}_{t}^{-1} \boldsymbol{\Sigma}_{t}$ for $h \geq 0$ is a $(n \times n)$ impulse response matrix; $\boldsymbol{\epsilon}_{t} \sim \mathbf{N}\left(\mathbf{0}, \mathbf{I}_{n}\right)$ is the stadardized structural shock vector; and note that $\boldsymbol{\Phi}_{h, t}=\mathbf{K M}_{t}^{h} \mathbf{K}^{\prime}$ denotes the $h^{\text {th }}$ matrix lag of the $\operatorname{VMA}(\infty)$ representation. More neatly, the orthogonalized impulse response function can be represented as

$$
\frac{\partial \mathbf{y}_{t}(h)}{\partial \boldsymbol{\epsilon}_{t}}=\boldsymbol{\Xi}_{t}(h), \quad h=0,1,2, \cdots
$$


where

$$
\boldsymbol{\Xi}_{t}(h)=\left[\begin{array}{cccc}
\Xi_{11, t}(h) & \Xi_{12, t}(h) & \cdots & \Xi_{1 n, t}(h) \\
\Xi_{21, t}(h) & \Xi_{22, t}(h) & \cdots & \Xi_{2 n, t}(h) \\
\vdots & \vdots & \ddots & \vdots \\
\Xi_{n 1, t}(h) & \Xi_{n 2, t}(h) & \cdots & \Xi_{n n, t}(h)
\end{array}\right]
$$

such that $\Xi_{i j}(h)$ denotes the response of variable $i$ to a shock in variable $j$ after $h$ periods. Note that with Cholesky factorization of the reduced form covariance matrix, the upper triangular elements in $\boldsymbol{\Xi}_{t}$ are zero for $h=0$. Likewise, the simultaneous response of a variable $i$ to shock in variable $j$ is, characterized by the elements of $\mathbf{A}_{t}^{-1} \boldsymbol{\Sigma}_{t}$.

The impulse response functions are computed for every MCMC draw by applying the posterior mean of the standard deviation over the sample period, $\bar{\sigma}_{i}=1 /(T-P) \sum_{t=P+1}^{T} \exp \left(\log \sigma_{i, t}^{2} / 2\right)$, at each sample period. This treatment of the impulse response allows the comparability across time, which is required since $\boldsymbol{\Sigma}_{t}$ is time varying. Finally, the impulse responses are computed with the assumption that VAR parameters remain constant at their current values, which corroborate with the notion of bounded rationality and learning, as stressed by Cogley et al. (2010).

\section{S.5.4 Computing predictability and persistence}

Following Benati and Surico (2008), we interpret the statistic proposed by Cogley et al. (2010) as the measure of predictability and the statistic by Cogley and Sargent (2005) as the measure of persistence.

\section{Predictability of a variable}

Following Cogley et al. (2010), predictability in the paper is defined as one minus the ratio of conditional variance (forecast error variance) to unconditional variance

$$
R_{q, t}^{2}(H)=1-\frac{\mathbf{k}_{q}^{\prime}\left[\sum_{h=0}^{H-1} \boldsymbol{\Phi}_{h, t} \boldsymbol{\Omega}_{t} \boldsymbol{\Phi}_{h, t}^{\prime}\right] \mathbf{k}_{q}}{\mathbf{k}_{q}^{\prime}\left[\sum_{h=0}^{\infty} \boldsymbol{\Phi}_{h, t} \boldsymbol{\Omega}_{t} \boldsymbol{\Phi}_{h, t}^{\prime}\right] \mathbf{k}_{q}},
$$

where $\mathbf{k}_{q}$ is a selection vector. The predictability measure we refer to here measures the 'gap' predictability, that is, the predictability of the difference from the observed value to the unconditional mean.

\section{Persistence of a variable}

The degree of persistence of a time series variable $q_{t}$ is measured, following Cogley and Sargent (2005), via the normalized spectrum on the frequency domain. The statistic is defined as the ratio 
of the spectrum for $q_{t}$ over the unconditional variance

$$
g_{q, t}(\omega)=\frac{f_{q, t}(\omega)}{\int_{-\pi}^{\pi} f_{q, t}(\omega) \mathrm{d} \omega},
$$

where

$$
f_{q, t}(\omega)=\frac{1}{2 \pi} \sum_{h=-\infty}^{\infty} \exp (i h \omega) \boldsymbol{\Gamma}_{q, t}(h)
$$

which can be rewritten as

$$
f_{q, t}(\omega)=\frac{1}{2 \pi}\left[\boldsymbol{\Gamma}_{q, t}(0)+2 \sum_{h=1}^{\infty} \boldsymbol{\Gamma}_{q, t}(h) \cos (h \omega)\right],
$$

using Euler's formula $\exp (i \omega)=\cos (\omega)+i \sin (\omega)$ and the fact that the spectrum is symmetric with $\cos (\omega)=\cos (-\omega)$ and $\boldsymbol{\Gamma}_{q, t}(h)=\boldsymbol{\Gamma}_{q, t}(-h)$ given covariance stationarity of the process.

The denominator of equation (S.5) is the unconditional variance of $q_{t}$

$$
\boldsymbol{\Gamma}_{q, t}(0)=\int_{-\pi}^{\pi} f_{q, t}(\omega) \mathrm{d} \omega=2 \int_{0}^{\pi} f_{q, t}(\omega) \mathrm{d} \omega
$$

by symmetry, implying that the statistic is normalized to allow for a better capture of the degree of persistence given time varying nature of the variance in the VAR. Moreover, invoking the inverse Fourier transform, $g_{q, t}(\omega)$ can be interpreted as a spectral density function since it integrates to one when $h=0$

$$
1=\rho_{q, t}(h)=\int_{-\pi}^{\pi} \exp (i h \omega) g_{q, t}(\omega) \mathrm{d} \omega
$$

There are at least two ways to estimate the spectrum. One way to estimate the spectrum in equation (S.6) is via the sample periodogram (explained in detail in Hamilton (1994))

$$
\begin{aligned}
\widetilde{f}_{q, t}(\omega) & =\frac{1}{2 \pi} \sum_{h=-T+1}^{T-1} \exp (i h \omega) \boldsymbol{\Gamma}_{q, t}(h), \\
& =\frac{1}{2 \pi}\left[\boldsymbol{\Gamma}_{q, t}(0)+2 \sum_{h=1}^{T-1} \boldsymbol{\Gamma}_{q, t}(h) \cos (h \omega)\right],
\end{aligned}
$$

where twice its ratio to the population spectrum has the asymptotic distribution

$$
\frac{2 \widetilde{f}_{q, t}(\omega)}{f_{q, t}(\omega)} \sim \chi^{2}(2)
$$

such that $\mathbb{E}\left[2 \widetilde{f}_{q, t}(\omega) / f_{q, t}(\omega)\right]=2$, implying that sample periodogram can provide an unbiased estimate of the population spectrum when $T$ is sufficiently large, i.e., $\lim _{T \rightarrow \infty} \mathbb{E}\left[\widetilde{f}_{q, t}(\omega)\right]=f_{q, t}(\omega)$. However, since we have

$$
\lim _{T \rightarrow \infty} \operatorname{Var}\left[\widetilde{f}_{q, t}(\omega)\right] \rightarrow\left\{\begin{array}{lll}
2 f_{q, t}^{2}(0) & \text { if } & \omega=0 \\
f_{q, t}^{2}(\omega) & \text { if } & \omega \neq 0
\end{array}\right.
$$

one must resort to nonparametric estimation of the population spectrum by introducing a kernel function, $\kappa_{h}^{*}$, into the periodogram. 
Here, we employ the modified Bartlett kernel of the form

$$
\kappa_{h}^{*}=\left\{\begin{array}{lll}
1-\frac{h}{H+1} & \text { for } & h=1,2, \cdots, H \\
0 & \text { for } & h>H
\end{array}\right.
$$

Thus, the Bartlett estimate of the spectrum is

$$
\widetilde{f}_{q, t}(\omega)=\frac{1}{2 \pi}\left[\boldsymbol{\Gamma}_{q, t}(0)+2 \sum_{h=1}^{H}\left(1-\frac{h}{H+1}\right) \boldsymbol{\Gamma}_{q, t}(h) \cos (h \omega)\right] .
$$

We set the smoothing parameter $H$ such that we compute the same low frequency region, $0 \leq \omega \leq$ $2 \pi h / T$, where $2 \pi h / T \approx 0.5$ as in Cogley and Sargent (2005).

Another way to estimate the spectrum is via what Cogley and Sargent (2005) call the local linear approximation

$$
f_{q, t}(\omega)=\mathbf{k}_{q}^{\prime}\left(\mathbf{\Upsilon}_{t}^{-1}\right) \frac{\boldsymbol{\Omega}_{t}}{2 \pi}\left(\mathbf{\Upsilon}_{t}^{-1}\right)^{*} \mathbf{k}_{q}
$$

where $\mathbf{k}_{q}$ is the selection column vector; $\left(\mathbf{\Upsilon}_{t}^{-1}\right)^{*}$ denotes the conjugate transpose of the inverse of $\mathbf{\Upsilon}_{t}=\mathbf{I}_{n}-\boldsymbol{\Phi}_{h, t} \exp (-i \omega)$ for $h=1$. In the main text, we report persistence statistic where the spectrum is computed as in Cogley and Sargent (2005), but we confirm that both the methods yield similar results.

\section{S.6 Additional results}

Figure S.2 plots the impulse responses of investment growth to an average-sized uncertainty shock over 20 quarters at three different points in time: 1985:I, 2000:I and 2010:I. The figure shows that investment growth declines significantly and follows a hump-shaped path. The "volatility overshoot" effect of uncertainty shock can be seen in the panel labelled 2000:I, where investment growth rebounds slightly after about a year following the initial decline. Figure S.3 confirms that such a "drop, rebound, and overshoot" effect coincides with a larger decline in the real interest rate, again as seen in the panel labelled 2000:I. This finding suggests that the rebound effect may be partly driven by endogenously expansionary monetary policy as argued in the text.

[Figure S.2 here]

[Figure S.3 here]

\section{S.7 Robustness checks}

In this section, we report results from alternative specifications of the time-varying VAR. The baseline specification is the specification reported in the manuscript and the robustness checks are conducted as departures from the baseline. Thus, unless otherwise stated, the baseline specification will be assumed. For convenience, we first describe the baseline specification before we move on. 


\section{The baseline model specification:}

The model is a standard time-varying VAR estimated with two lags. The structural shocks are identified based on recursive identification scheme. The baseline ordering of the endogenous variables are as follows

$$
\mathbf{y}_{t}=\left[s_{t}, \pi_{t}, i_{t}, r_{t}\right]^{\prime}
$$

where, $s_{t}$ is the uncertainty proxy, $\pi_{t}$ stands for the annualized quarter-on-quarter inflation rate, $i_{t}$ is the annualized quarter-on-quarter growth rate of investment, and $r_{t}$ is the ex-post real interest rate..

The following priors are considered as a baseline

$$
\begin{aligned}
\boldsymbol{\beta}_{P+1} & \sim \mathrm{N}(\mathbf{0}, 10 \mathbf{I}), & Q_{i} & \sim \mathrm{G}^{-1}(40 / 2,0.004 / 2), \\
\boldsymbol{\alpha}_{P+1} & \sim \mathrm{N}(\mathbf{0}, 10 \mathbf{I}), & S_{i} & \sim \mathrm{G}^{-1}(12 / 2,0.01 / 2), \\
\log \boldsymbol{\sigma}_{P+1}^{2} & \sim \mathrm{N}(\mathbf{0}, 10 \mathbf{I}), & W_{i} & \sim \mathrm{G}^{-1}(12 / 2,0.01 / 2),
\end{aligned}
$$

where, $\mathrm{N}$ and $\mathrm{G}^{-1}$ respectively denote the Gaussian and inverse Gamma distributions. With this set up, the model is estimated using the MCMC method outlined in $\S$ S.3.

On top of the sensitivity analyses in the text, we conduct the following additional robustness checks with respect to: i) alternative proxy for uncertainty, ii) prior sensitivity, iii) different ordering of the endogenous variables in the time-varying VAR, and iv) different lag length. Each of the following subsection presents a brief explanation of the specific changes made relative to the baseline, as well as the accompanying results.

\section{S.7.1 Alternative proxy for uncertainty}

Uncertainty is difficult to quantify and as a result the empirical literature has proposed several uncertainty proxies. Rather than taking a stand on a particular proxy, here we consider three different proxies, which roughly span the range of approaches used to infer fluctuations in financial uncertainty.

Our first uncertainty proxy is based on stock market volatility as in the main text, i.e. the 30-day implied volatility of the S\&P100 index (VXO). Since the index is unavailable before 1986, pre-1986 monthly return volatilities are computed by employing the monthly standard deviation of the daily S\&P500 index as before (normalized to same mean and variance as the VXO index when they overlap from 1986 onward). Our second uncertainty proxy is the one proposed by Gilchrist et al. (2014) and captures common shocks in the idiosyncratic volatility of equity returns (GSZ). Finally, we also consider the financial uncertainty index proposed by Jurado et al. (2015), which measures a common component in the time-varying volatilities of h-step ahead forecast errors across a large number of financial series (JLN). ${ }^{3}$

We reduce the dimensions of these three proxies via the principal component method. This

\footnotetext{
${ }^{3}$ We use the JLN measure of financial uncertainty at the 12-month forecast horizon, however, results are robust to shorter horizons.
} 
treatment enabls the extraction of the most important element describing the three processes. We compute the first principal component from the (demeaned and standardized) monthly uncertainty proxies. The resulting series is then demeaned and standardized again before it is averaged within the quarter.

Figure S.4 plots the uncertainty proxy based on stock market volatility used in the main text and the first principal component (FPC) extracted from the three series. As seen in the figure, they are very similar with a correlation of 0.95 . Figure S.5 shows the impulse responses to a normalized uncertainty shock when using the FPC as a proxy for uncertainty, which is again very similar to the impulse responses shown in the text.

[Figure S.4 here]

[Figure S.5 here]

\section{S.7.2 Prior sensitivity}

In the robustness checks performed in the text involving five-variate VARs, priors were set such that we allowed for relatively less movements in the VAR coefficients relative to the baseline priors, in order to render the VARs stable. Specifically, the following priors were used:

$$
\begin{array}{rlrl}
\boldsymbol{\beta}_{P+1} & \sim \mathrm{N}(\mathbf{0}, 10 \mathbf{I}), & Q_{i} & \sim \mathrm{G}^{-1}(80 / 2,0.002 / 2), \\
\boldsymbol{\alpha}_{P+1} & \sim \mathrm{N}(\mathbf{0}, 10 \mathbf{I}), & S_{i} \sim \mathrm{G}^{-1}(12 / 2,0.01 / 2), \\
\log \boldsymbol{\sigma}_{P+1}^{2} \sim \mathrm{N}(\mathbf{0}, 10 \mathbf{I}), & W_{i} \sim \mathrm{G}^{-1}(12 / 2,0.01 / 2),
\end{array}
$$

Figure S.6 depicts the impulse responses to a normalized uncertainty shock and we confirm that all our baseline results remain robust.

[Figure S.6 here]

\section{S.7.3 Different ordering}

Here, we provide results with a different ordering of the endogenous variables in the time-varying VAR, with the priors the same as in the manuscript. Specifically, the following ordering is considered: $\mathbf{y}_{t}=\left[s_{t}, \pi_{t}, r_{t}, i_{t}\right]^{\prime}$. This ordering makes investment the most endogenous variable in the model by also allowing it to respond to changes in the real interest rate. The results remain robust and below we show the impulse responses under the above ordering.

[Figure S.7 here] 


\section{S.7.4 Different lag length}

Following Cogley and Sargent (2005) and Primiceri (2005), we set the lag length to two in the baseline estimation. Here, we provide results from the time-varying VAR with three lags. We report the impulse responses below in Figure S.8 with three lags.

[Figure S.8 here] 
Figures

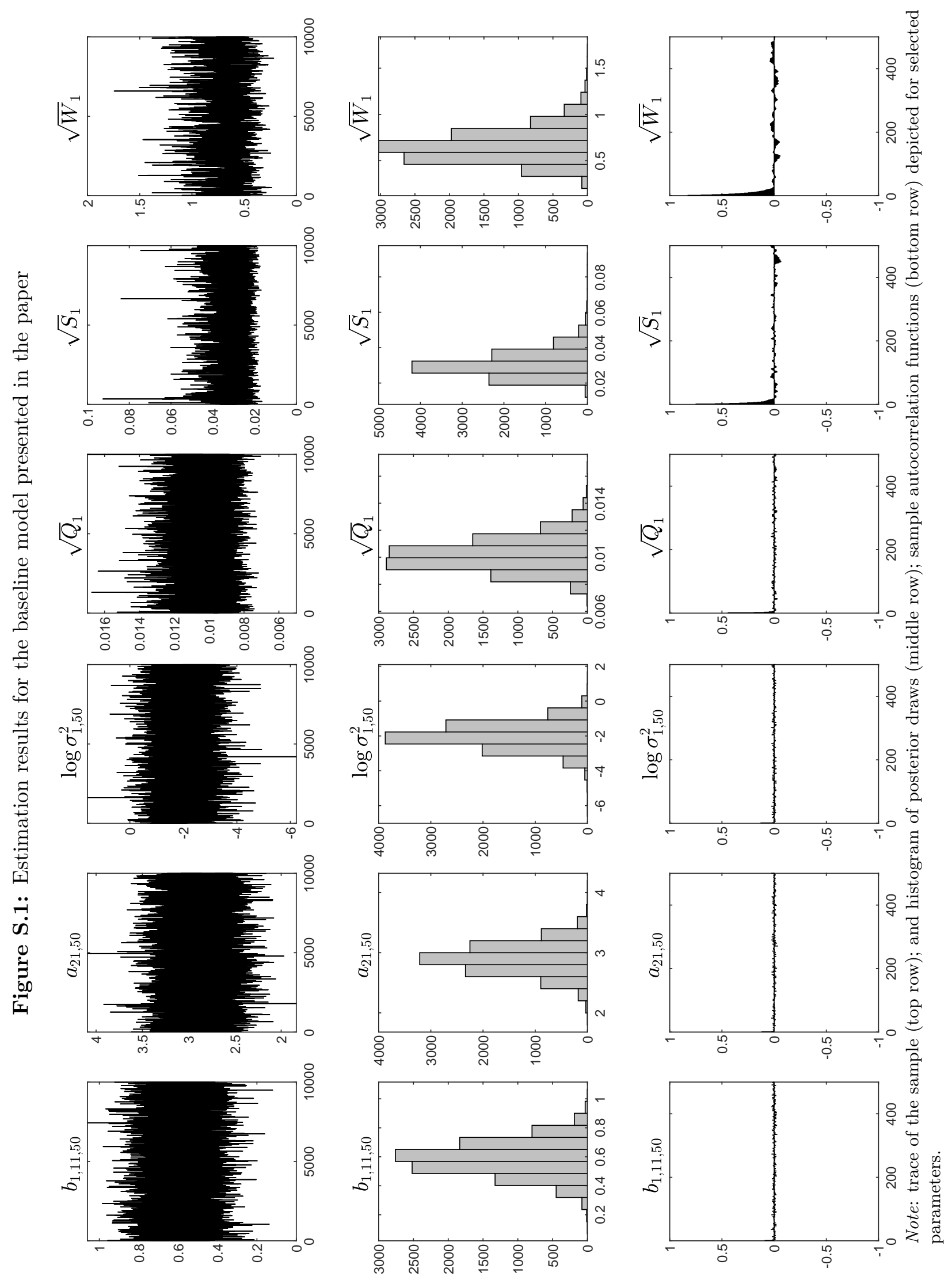


Figure S.2: Response of investment growth to a normalized uncertainty shock
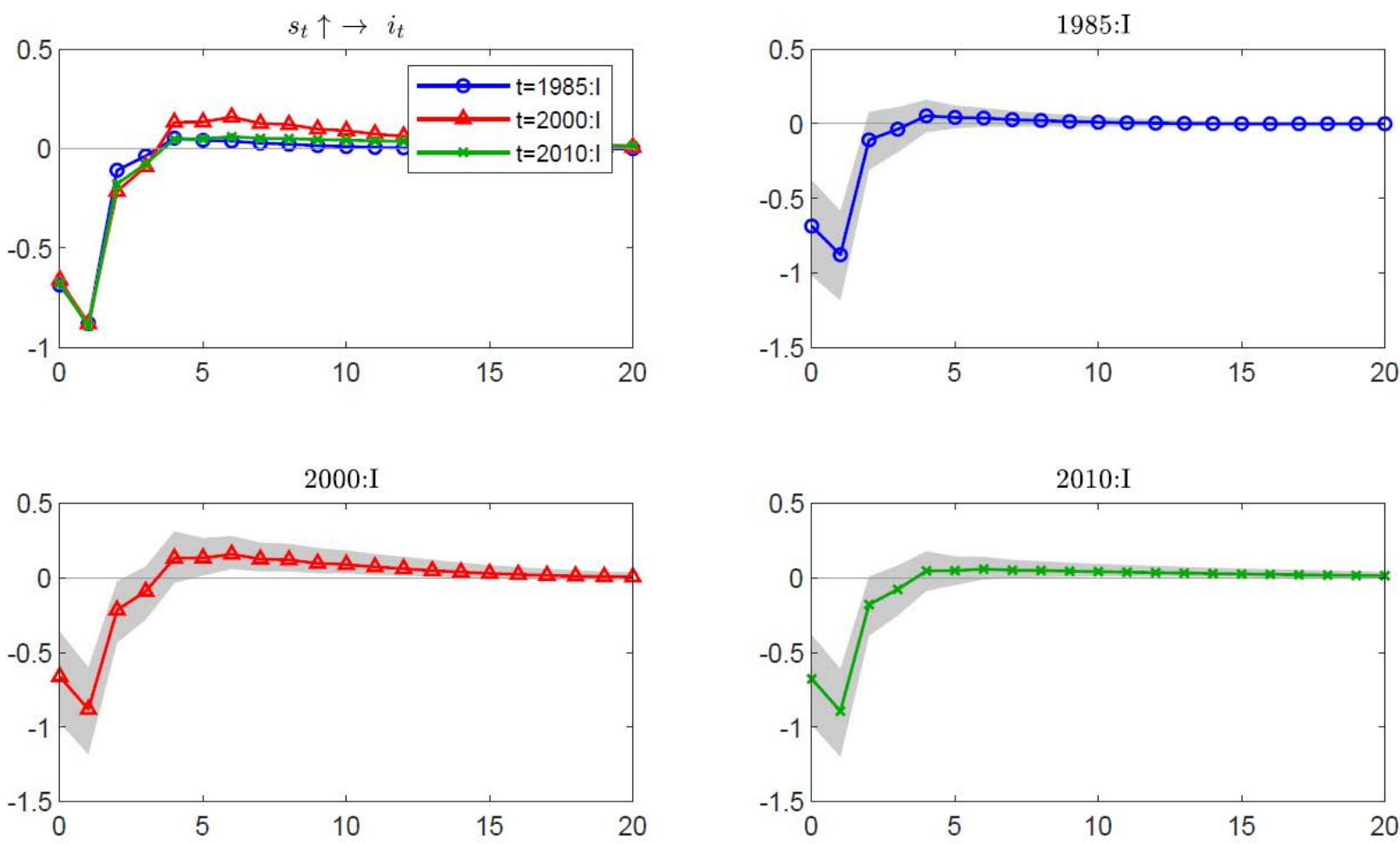

Note: The baseline identification ordering $\mathbf{y}_{t}=\left[s_{t}, \pi_{t}, i_{t}, r_{t}\right]^{\prime}$ adopted. The panels show the impulse response of investment growth $i_{t}$ to an average-sized uncertainty shock $s_{t}$ over 20 quarters in three different time periods: 1985:I, 2000:I and 2010:I. The gray shaded regions represent the $68 \%$ posterior credible intervals around the posterior median depicted in solid lines. 
Figure S.3: Response of real interest rate to a normalized uncertainty shock
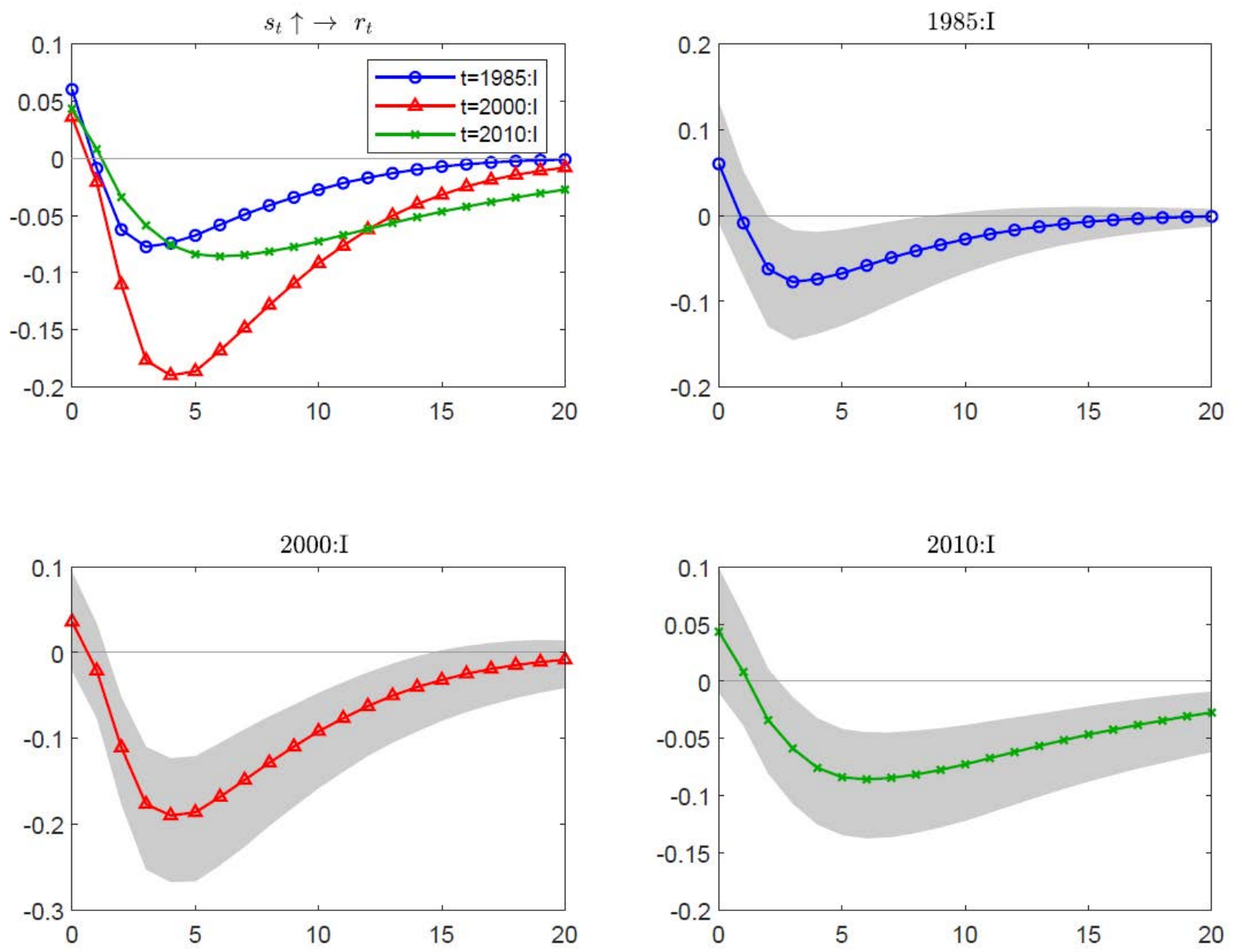

Note: The baseline identification ordering $\mathbf{y}_{t}=\left[s_{t}, \pi_{t}, i_{t}, r_{t}\right]^{\prime}$ adopted. The panels show the impulse response of the real interest rate $r_{t}$ to an average-sized uncertainty shock $s_{t}$ over 20 quarters in three different time periods: 1985:I, 2000:I and 2010:I. The gray shaded regions represent the $68 \%$ posterior credible intervals around the posterior median depicted in solid lines. 
Figure S.4: Uncertainty Proxy

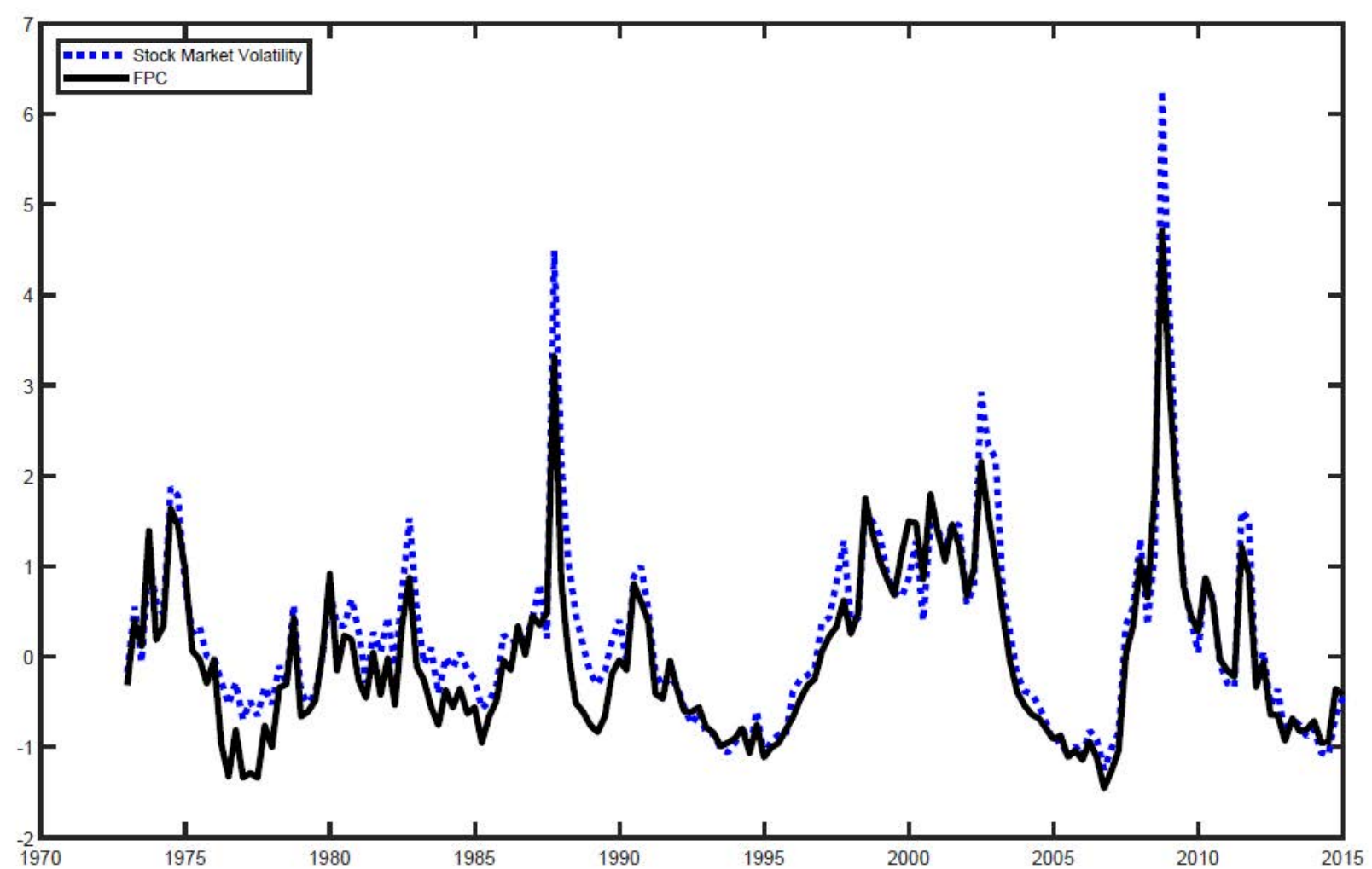

Note: Data spans from 1973:I to 2015:I (based on data availability), averaged from monthly to quarterly frequency. FPC denotes the first principal component of the three uncertainty proxies. Both measures are demeaned and standardized. 
Figure S.5: Impulse responses to a normalized uncertainty shock - Alternative Uncertainty Proxy
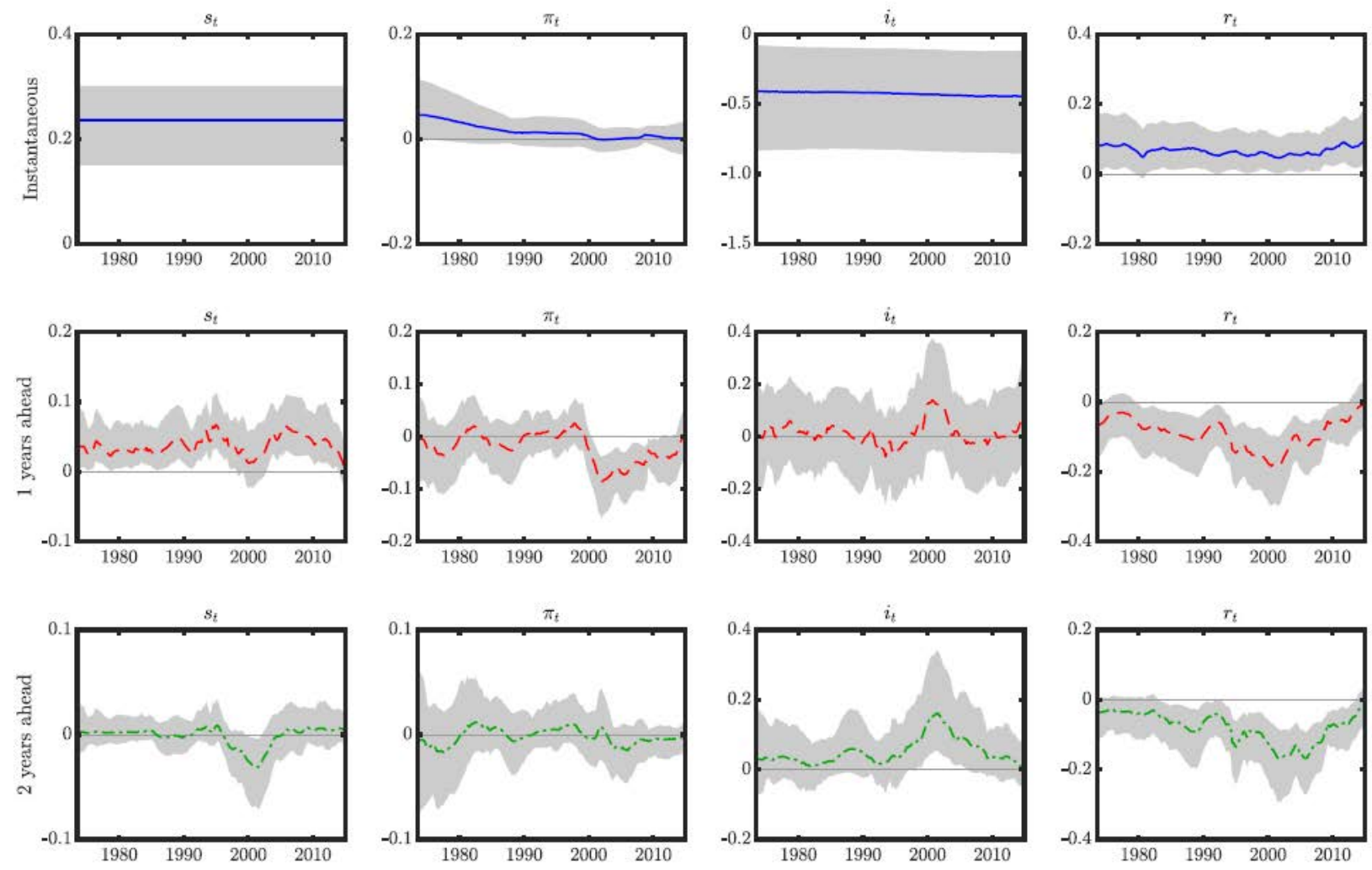

Note: Instantaneous, 1-year and 2-year ahead impulse responses. The identification ordering $\mathbf{y}_{t}=\left[s_{t}, \pi_{t}, i_{t}, r_{t}\right]^{\prime}$ adopted. The first column shows the response of $s_{t}$ : uncertainty, second column shows the response of $\pi_{t}$ : inflation, third column shows the response of $i_{t}$ : investment growth and the fourth column shows the response of $r_{t}$ : real interest rate. The gray shaded regions represent the $68 \%$ posterior credible intervals around the posterior median depicted in solid, dashed, and dashed-dotted lines. 
Figure S.6: Impulse responses to a normalized uncertainty shock - Prior sensitivity
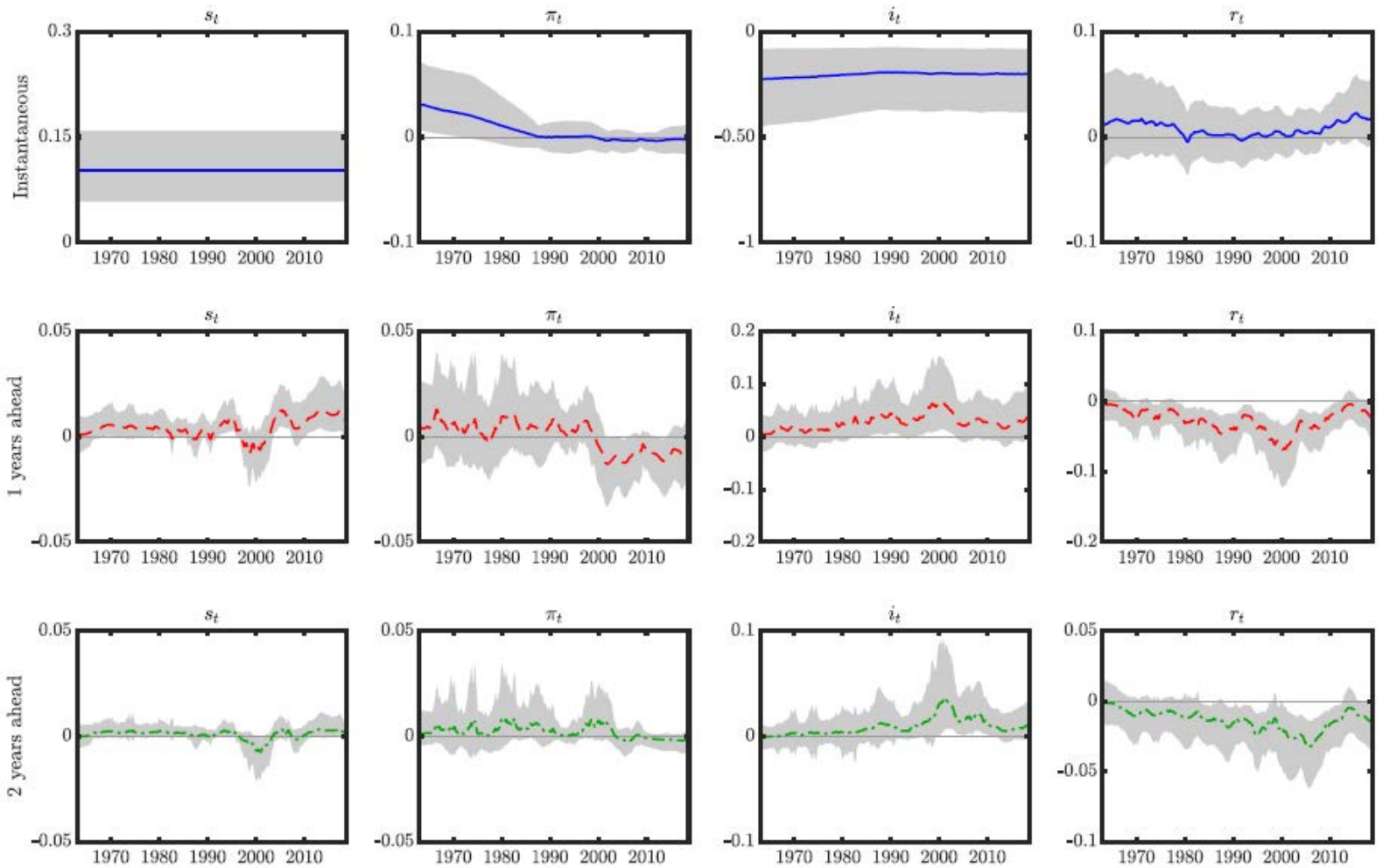

Note: Instantaneous, 1-year and 2-year ahead impulse responses. The identification ordering $\mathbf{y}_{t}=\left[s_{t}, \pi_{t}, i_{t}, r_{t}\right]^{\prime}$ adopted. The first column shows the response of $s_{t}$ : uncertainty, second column shows the response of $\pi_{t}$ : inflation, third column shows the response of $i_{t}$ : investment growth and the fourth column shows the response of $r_{t}$ : real interest rate. The gray shaded regions represent the $68 \%$ posterior credible intervals around the posterior median depicted in solid, dashed, and dashed-dotted lines. 
Figure S.7: Impulse responses to a normalized uncertainty shock - Different ordering
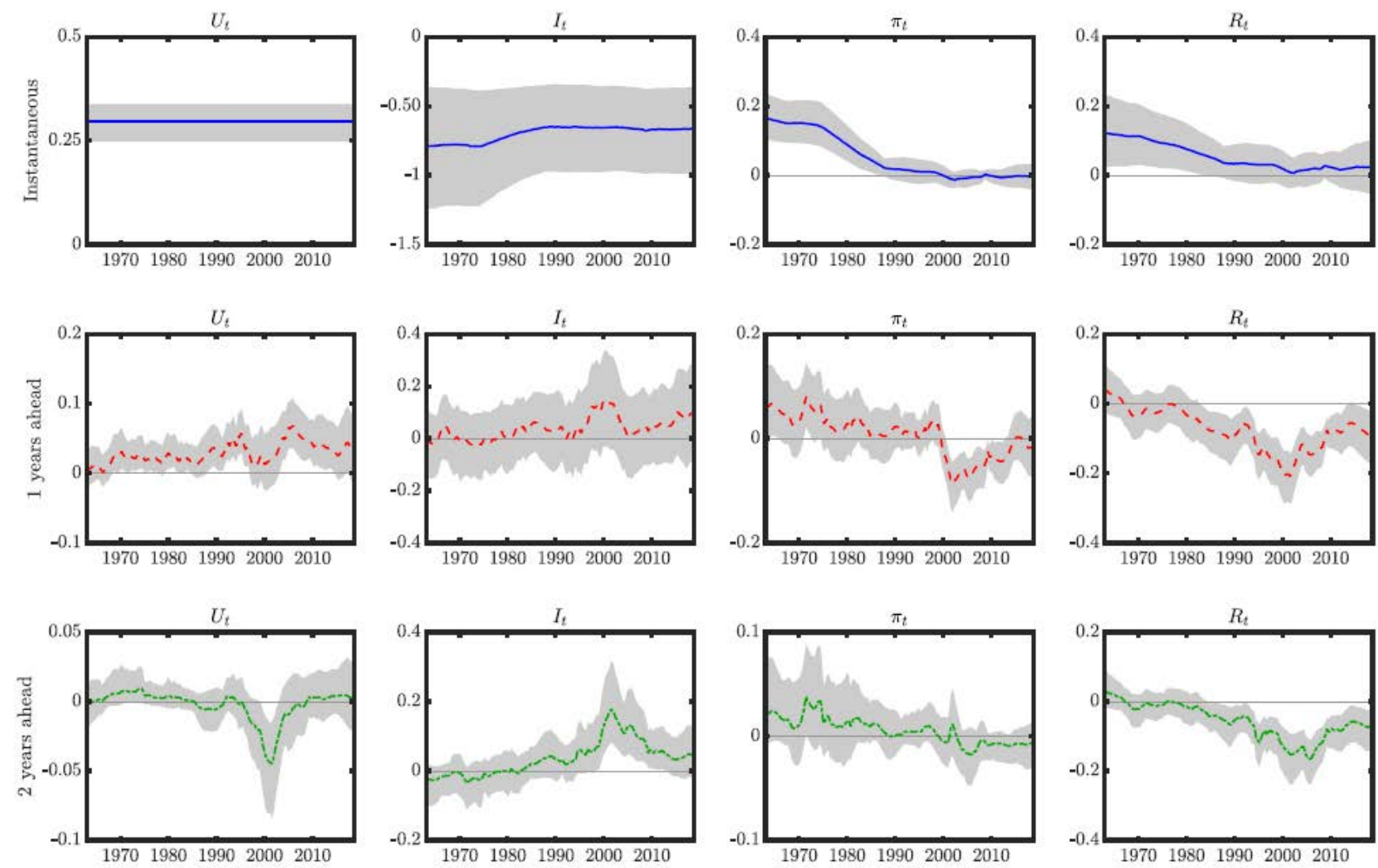

Note: Instantaneous, 1-year and 2-year ahead impulse responses. The identification ordering $\mathbf{y}_{t}=\left[s_{t}, \pi_{t}, r_{t}, i_{t}\right]^{\prime}$ adopted. The first column shows the response of $s_{t}$ : uncertainty, second column shows the response of $\pi_{t}$ : inflation, third column shows the response of $i_{t}$ : investment growth and the fourth column shows the response of $r_{t}$ : real interest rate. The gray shaded regions represent the $68 \%$ posterior credible intervals around the posterior median depicted in solid, dashed, and dashed-dotted lines. 
Figure S.8: Impulse responses to a normalized uncertainty shock - Lag Length
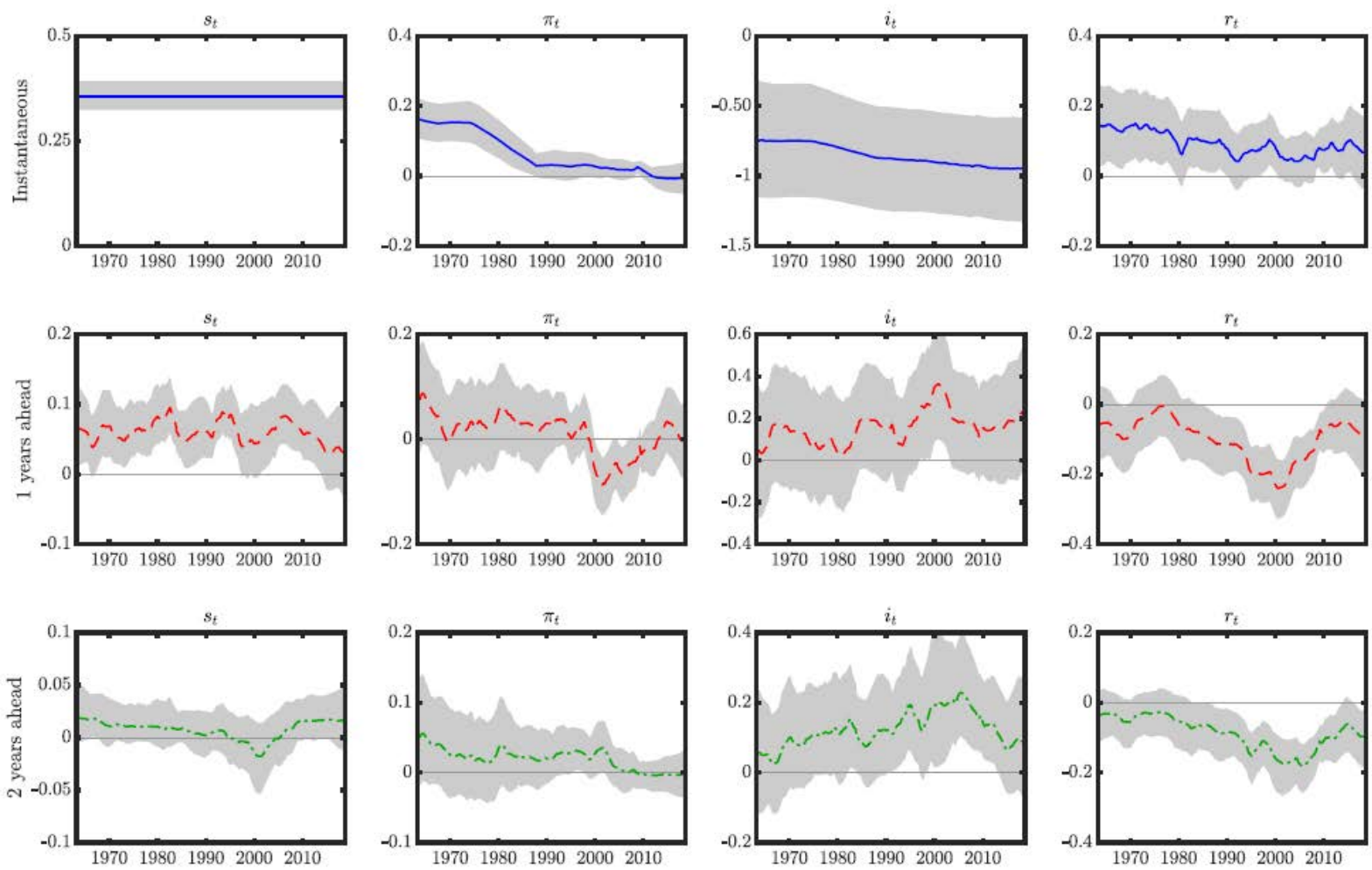

Note: Instantaneous, 1-year and 2-year ahead impulse responses. The identification ordering $\mathbf{y}_{t}=\left[s_{t}, \pi_{t}, i_{t}, r_{t}\right]^{\prime}$ adopted. VAR lag length is three. The first column shows the response of $s_{t}$ : uncertainty, second column shows the response of $\pi_{t}$ : inflation, third column shows the response of $i_{t}$ : investment growth and the fourth column shows the response of $r_{t}$ : real interest rate. The gray shaded regions represent the $68 \%$ posterior credible intervals around the posterior median depicted in solid, dashed, and dashed-dotted lines. 


\section{References}

Bachmann, R., Elstner, S., and Sims, E.R. (2013), "Uncertainty and Economic Activity: Evidence from Business Survey Data", American Economic Journal: Macroeconomics 5 (2), pp. 217-249.

Benati, L. and Surico, P. (2008), "Evolving U.S. Monetary Policy and the Decline of Inflation Predictability", Journal of the European Economic Association 6 (2/3), pp. 634-646.

Beveridge, S. and Nelson, C.R. (1981), "A New Approach to Decomposition of Economic Time Series into Permanent and Transitory Components with Particular Attention to Measurement of the 'Business Cycle'”, Journal of Monetary Economics 7 (2), pp. 151-174.

Bloom, N. (2009), "The Impact of Uncertainty Shocks", Econometrica 77 (3), pp. 623-685.

Caldara, D., Fuentes-Albero, C., Gilchrist, S., and Zakrajšek, E. (2016), "The Macroeconomic Impact of Financial and Uncertainty Shocks", European Economic Review 88, pp. 185-207.

Cogley, T., Primiceri, G.E., and Sargent, T.J. (2010), "Inflation-Gap Persistence in the US", American Economic Journal: Macroeconomics 2 (1), pp. 43-69.

Cogley, T. and Sargent, T.J. (2001), "Evolving Post-World War II U.S. Inflation Dynamics", NBER Macroeconomics Annual 2001, ed. by B.S. Bernanke and K. Rogoff, vol. 16, Cambridge, MA: MIT Press, pp. 331-388.

- (2005), "Drift and Volatilities: Monetary Policies and Outcomes in the Post WWII US", Review of Economic Dynamics 8 (2), pp. 262-302.

de Jong, P. and Shephard, N. (1995), "The Simulation Smoother for Time Series Models", Biometrica 82 (2), pp. 339-350.

Gilchrist, S., Sim, J.W., and Zakrajšek, E. (2014), Uncertainty, Financial Frictions, and Investment Dynamics, Working paper 20038, NBER.

Hamilton, J.D. (1994), Time Series Analysis, Princeton University Press.

Jacquier, E., Polson, N.G., and Rossi, P.E. (1994), "Bayesian Analysis of Stochastic Volatility Models", Journal of Business 83 Economic Statistics 12 (4), pp. 371-389.

Jurado, K., Ludvigson, S.C., and Ng, S. (2015), "Measuring Uncertainty", American Economic Review 105 (3), pp. 1177-1216.

Justiniano, A., Primiceri, G.E., and Tambalotti, A. (2010), "Investment Shocks and Business Cycles", Journal of Monetary Economics 57 (2), pp. 132-145.

Kim, S., Shephard, N., and Chib, S. (1998), "Stochastic Volatility: Likelihood Inference and Comparison with ARCH Models", Review of Economic Studies 65 (3), pp. 361-393.

Lütkepohl, H. (2005), New Introduction to Multiple Time Series Analysis, Springer Science \& Business Media.

Nakajima, J. (2011), "Time-Varying Parameter VAR Model with Stochastic Volatility: An Overview of Methodology and Empirical Applications", Monetary and Economic Studies 29, pp. 107-142. 
Nakajima, J., Kasuya, M., and Watanabe, T. (2011), "Bayesian Analysis of Time-varying Parameter Vector Autoregressive Model for the Japanese Economy and Monetary Policy", Journal of the Japanese and International Economies 25 (3), pp. 225-245.

Primiceri, G.E. (2005), "Time Varying Structural Vector Autoregressions and Monetary Policy", Review of Economic Studies 72 (3), pp. 821-852.

Shephard, N. and Pitts, M. (1997), "Likelihood Analysis of Non-Gaussian Measurement Time Series", Biometrica 84 (3), pp. 653-667.

Smets, F. and Wouters, R. (2007), "Shocks and Frictions in US Business Cycles: A Bayesian DSGE Approach", American Economic Review 97 (3), pp. 586-606.

Watanabe, T. and Omori, Y. (2004), "A Multi-move Sampler for Estimating Non-Gaussian Time Series Models: Comments on Shephard and Pitt (1997)", Biometrica 91 (1), pp. 246-248.

Wu, J.C. and Xia, F.D. (2016), "Measuring the Macroeconomic Impact of Monetary Policy at the Zero Lower Bound", Journal of Money, Credit and Banking 48 (2-3), pp. 253-291. 